\title{
An investigation on a supercritical aerofoil with a wavy leading edge in a transonic flow
}

\author{
Enrico Degregori, a) and Jae Wook Kim ${ }^{1, b}$ \\ Institute of Sound and Vibration Research, University of Southampton, Southampton, SO17 1BJ, \\ United Kingdom
}

(Dated: 22 June 2020)

A compressible large-eddy simulation is performed to study the effects of a wavy leading edge (WLE) applied on a supercritical aerofoil in a transonic flow at $R e_{\infty}=5.0 \times 10^{5}$ and $M_{\infty}=0.7$. The wavelength and peak-to-trough amplitude of the WLE used in this study are $5 \%$ and $2.5 \%$, respectively, of the mean aerofoil chord. The primary aim of the study is to understand the aerodynamic characteristics of the modified aerofoil over a range of incidence angles. For this reason, a slow heaving motion is imposed where the geometric angle of attack is gradually increased from $\alpha_{g}=2^{\circ}$ to $7^{\circ}$ without a significant dynamic (added mass) effect, i.e. a quasi-linear range. The new transonic flow study shows significantly different findings (with some similar features) to the previous low-speed flow studies. It is observed in the quasi-linear range that the modified aerofoil achieves a performance improvement at low and moderate angles because of a drag reduction in the leading edge region and downstream of the laminar-turbulent (L-T) transition point. The leading edge (LE) analysis shows that the maximum pressure coefficient remains equal to that of the baseline case only at the trough and peak sections. The relative decrease of pressure at the LE results in the drag reduction. The transonic flow at the LE is analysed in further details to show a reversed flow region at the trough and its influence on the boundary layer development over the aerofoil. Also, the spanwise variation of the boundary layer characteristics over the modified aerofoil is evaluated and analysed. One of the most notable findings in this paper is that the flow at the trough becomes supersonic even at low angles of attack and this results in an enhanced LE flow acceleration spread across the span, which seems facilitated by using a short WLE wavelength. This flow behaviour is qualitatively explained by using an analogy between a channeling effect and a convergent-divergent nozzle in a transonic flow. Another notable observation is that there is an upstream movement of the laminar-turbulent transition point seemingly related to the flow distortion around the WLE. Interestingly, the flow distortion introduces a three dimensionality into the laminar boundary layer but it keeps the flow laminar, so the benefits of the laminar supercritical aerofoil are not lost. These LE phenomena have a major impact on the shock structure at high incidence angles where the more energetic laminar boundary layer changes the shock structure over the modified aerofoil. This can be crucial to control the shock buffet phenomenon.

\section{INTRODUCTION}

Aircraft flight in a transonic speed regime represents a huge challenge in terms of fuel consumption, fluid-structure interaction and noise emission. The reason is related to a decreased efficiency caused by a drag divergence and to an unsteady loading because of shock wave oscillations. Some supercritical aerofoils to alleviate these issues can be found in commercial aviation aircrafts wings, helicopters rotor blades and compressor blades. In addition, control devices exist to improve the aerodynamic performance in transonic flow, which can be classified into three main categories: trailing edge devices (TEDs), vortex generators and shock control bumps.

TEDs were originally studied for shock oscillation control because a common feature among the classic models governing the buffet phenomenon is the coupling between the large scales excursions of the shock and the pressure fluctuations at the trailing edge. Static trailing edge flap deflections can modify the flow field and stabilise it. In transonic flows they can diminish significantly the buffet lift response, indicating that the aerodynamic coefficients can be controlled by prescribed structural motions at this condition 1 . The drawbacks are drag penalties and longitudinal instability, but they can be tackled with a feedback loop control2 . Open loop static TEDs deflections delay the onset of buffet to higher lift coefficients, but the control strategy is not effective in the developed buffet region at reducing the magnitude of pressure fluctuations. The efficacy is increased with close loop control, but the performance decreases with three dimensional wing-fuselage model $\underline{3}$.

Mechanical and fluidic vortex generators (VGs) have been studied extensively to understand their effect on shock wave boundary layer interaction (SBLI), which is the driving phenomenon of shock oscillation 4 - Vortex generators

\footnotetext{
a) Electronic mail: E.Degregori@soton.ac.uk

b) Electronic mail: j.w.kim@soton.ac.uk
} 
energise and stabilise the boundary layer, promoting attached flow and inhibiting shock induced separation which is a key feature of buffet phenomenon. Previous studies ${ }^{7}$ show that VGs over an aerofoil improve the aerodynamic performance at incidence close to buffet onset with the downside of drag penalties addition at lower angles of attack. In particular, the shock wave is moved downstream over the modified aerofoil and the effect is increased with fluidic VGs. As long as the vortex generators are positioned upstream of the shock, the chordwise location has a negligible influence on the aerodynamic performance ${ }^{9}$. When the VGs are installed on a three dimensional wing with a sweep angle, the wing displays more sensitivity to installation effects due to cross flow additional component ${ }^{10 \mid 11}$. The open loop fluidic vortex generators can adopt a continuous or a pulsed approach. The mass flow rate is the main parameter of the continuous VGs and it is observed that the flow rate effects saturate above a threshold $\left(C_{\mu} \geq 5 \times 10^{-4}\right)^{8}$. The pulsated VGs are influenced by the forcing frequency. Low frequency pulsing results in shock smearing, while high frequency pulsing stabilises the shock and locates it upstream relative to the continuous fluidic blowing.

Two dimensional shock control bumps (SCBs) were originally studied for pressure wave drag reduction 12113 . The working principle is the split of a normal shock into a $\lambda$-shape shock because, in this way, the total pressure losses are decreased. SCBs work properly in design condition but they perform poorly off design. When the shock moves upstream, a second normal shock is generated by the bump. On the other hand, once the shock moves downstream, the separation increases and the boundary layer thickens. An experimental study 13 has proved that a sweep angle has a small influence on the bump effectiveness. Both pressure distribution and boundary layer development are similar over an aerofoil and a swept wing. The drag reduction by the bump is slightly lower for the swept case due to the lower contribution of wave drag to total drag. Three dimensional bumps have the same working principle for wave drag reduction $\frac{14}{14}$. A good performance is observed over unswept wings, while a sweep angle diminishes the efficiency $\frac{15}{15}$.

More recent studies consider SCBs applied to shock buffet contro 16117 . Two dimensional SCBs can delay transonic buffet onset by introducing a region of attached flow between the shock wave and the trailing edge of a supercritical

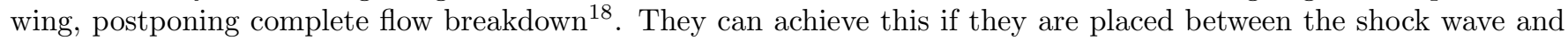
the wing trailing edge, where they do not offer any drag benefit. Three dimensional bumps applied to shock oscillation control have a different working principle because they generate two streamwise vortices adjacent to the bump which energise the boundary layer. They operate as 'smart' vortex generators 17. For this reason, their design position is more upstream than the two dimensional ones. Their performance is still not well defined; previous studies show that it can suppress the shock oscillation ${ }^{16}$ or decrease the angle of buffet onset $\frac{19}{}$. The main issue is that the optimal position for wave drag reduction is always different from the one for shock buffet suppression. A trade-off is needed to improve the overall mean and unsteady characteristics of an aerofoil 17 .

Wavy leading edges can be described as 'smart' vortex generators because they create counter-rotating streamwise vortices at the trough ${ }^{20121}$, without introducing discontinuity in the aerofoil geometry. It has been observed ${ }^{22}$ that streamwise BL streaks can attenuate disturbances and delay the L-T transition. This can have a beneficial effect on laminar supercritical aerofoils and improve the aerodynamic characteristics in transonic flows. Previous applications of this passive technique are limited to low speed flows, usually $M_{\infty} \leq 0.3$. WLE or tubercles aerofoils have been aerodynamically studied in the past, mainly at high incidence angles. The maximum lift coefficient $\left(C_{L_{\max }}\right)$ decreases with leading edge tubercles but the post-stall characteristics are less abrupt than SLE aerofoils. Hence, they improve the efficiency $(L / D)$ in the post-stall regime, while at lower angles of attack the baseline aerofoil is generally more efficient 2325 . A recent study 20 has observed also a reduction of unsteady fluctuations in the aerodynamic force in the post-stall regime. The performance improvement is driven by the modified flow field at the leading edge. Previous studies $2012123 \sqrt[25]{ }$ agree that the flow tends to separate at the troughs, while it remains attached at the peaks. Separated flow in the trough area is followed by a reattachment further downstream forming a laminar separation bubble. At high angles of attack, some LSBs group together to form a large area of attached flow behind them. Another commonly observed feature in the study of WLEs is the counter rotating streamwise vortices which seem to originate from the trough are $2^{2123} 25$. Wavy geometry determines these vortical structures ${ }^{21}$. The WLE wavelength $\lambda_{L E}$ defines the interaction between vortices, while the amplitude $h_{L E}$ is similar to the vortex generator's height. The main parameter to characterise the vortical structures is the local sweep angle $h_{L E} / \lambda_{L E}$.

The present work is aimed to apply for the first time WLEs to a supercritical aerofoil in a transonic flow. The first question is, will the common trend observed in the low speeds (decreased efficiency at low angles and increased efficiency at high angles when wavy aerofoils are used) still remain consistent in the high speed? The second question is, how does the flow field change in terms of counter rotating vortices and LSBs in the transonic speed? Since answers to these fundamental questions have not previously been available, it is necessary to perform a preliminary study to observe and understand how the wavy aerofoil responds to a range of angles of attack. We propose to simulate a slowly heaving aerofoil for this purpose with a minimal effect of added mass (dynamic stall). The objective is to create a working frame for future studies at static incidence. This approach is driven by the fact that there is no knowledge about aerodynamic behaviour of WLEs in high speed flows and it would be computationally expensive to perform several different static simulations. The limitation of this strategy is that the unsteady characteristics of the aerofoils at a certain incidence angle may not be precisely determined. This restriction is accepted for now because 
the main goal is to investigate the overall aerodynamic performance, while some discussions about the unsteady load can be considered in a second step.

This paper is structured in the following order. \$II introduces the computational set-up and methods used in this study including details of the heaving motion. \$III provides the aerodynamic performance evaluation during the quasi-linear part of the full cycle. $\$ \mathrm{IV}$ analyses in detail the leading edge flow field and its effects on the downstream flow concentrating on the evolution of streamwise vortices behind the leading edge, the leading edge acceleration, the boundary layer thickness and the leading edge flow channeling. \$V investigates the shock boundary layer interaction relating the LE flow distortion with a different shock structure over the modified aerofoil. Finally, concluding remarks and future work plans are given in $\mathrm{VI}$.

\section{PROBLEM DESCRIPTION AND METHODOLOGY}

The full 3-D Navier-Stokes equations (with a moving frame term included) can be written in a conservative form, transformed onto a generalised coordinate system as

$$
\frac{\partial}{\partial t}\left(\frac{\boldsymbol{Q}}{J}\right)+\frac{\partial}{\partial \xi_{i}}\left(\frac{\boldsymbol{E}_{j}-\hat{u}_{j} \boldsymbol{Q}-R e_{\infty}^{-1} M_{\infty} \boldsymbol{F}_{j}}{J} \frac{\partial \xi_{i}}{\partial x_{j}}\right)=-\frac{a_{\infty}}{L_{c}} \frac{\boldsymbol{S}}{J}
$$

where the indices $i=1,2,3$ and $j=1,2,3$ denote the three dimensions. The vectors of the conservative variables, inviscid and viscous fluxes are given by

$$
\left.\begin{array}{c}
\boldsymbol{Q}=\left[\rho, \rho u, \rho v, \rho w, \rho e_{\mathrm{t}}\right]^{T}, \\
\boldsymbol{E}_{j}=\left[\rho u_{j},\left(\rho u u_{j}+\delta_{1 j} p\right),\left(\rho v u_{j}+\delta_{2 j} p\right),\left(\rho w u_{j}+\delta_{3 j} p\right),\left(\rho e_{\mathrm{t}}+p\right) u_{j}\right]^{T}, \\
\boldsymbol{F}_{j}=\left[0, \tau_{1 j}, \tau_{2 j}, \tau_{3 j}, u_{i} \tau_{j i}+q_{j}\right]^{T}
\end{array}\right\}
$$

with the stress tensor and heat flux vector written as

$$
\tau_{i j}=\mu\left(\frac{\partial u_{i}}{\partial x_{j}}+\frac{\partial u_{j}}{\partial x_{i}}-\frac{2}{3} \delta_{i j} \frac{\partial u_{i}}{\partial x_{i}}\right), \quad q_{j}=\frac{\mu}{(\gamma-1) \operatorname{Pr}} \frac{\partial T}{\partial x_{j}}
$$

where $\xi_{i}=\{\xi, \eta, \zeta\}$ are the generalised coordinates, $x_{j}=\{x, y, z\}$ are the Cartesian coordinates, $\delta_{i j}$ is the Kronecker delta, $u_{j}=\{u, v, w\}, e_{\mathrm{t}}=p /[(\gamma-1) \rho]+u_{j} u_{j} / 2$ and $\gamma=1.4$ for air. The extra term $\hat{u}_{j} \boldsymbol{Q}$ is in place to implement the moving frame (heaving motion) for the present simulation (to be detailed). The local dynamic viscosity $\mu$ is calculated by using Sutherland's law 26 . In the current set-up, $\xi, \eta$ and $\zeta$ are aligned in the streamwise, vertical and lateral directions, respectively. The Jacobian determinant of the coordinate transformation (from Cartesian to the generalised) is given by $J^{-1}=|\partial(x, y, z) / \partial(\xi, \eta, \zeta)|^{27}$. The extra source term $\boldsymbol{S}$ on the right-hand side of Eq. 1 is non-zero within the sponge layer only, which is described in Kim, Lau, and Sandham 28.29]. In this paper, the freestream Mach and Reynolds numbers are defined as $M_{\infty}=U_{\infty} / a_{\infty}$ and $R e_{\infty}=\rho_{\infty} U_{\infty} L_{c} / \mu_{\infty}$ where $a_{\infty}=\sqrt{\gamma p_{\infty} / \rho_{\infty}}$ is the ambient speed of sound and $U_{\infty}=\sqrt{u_{\infty}^{2}+v_{\infty}^{2}+w_{\infty}^{2}}$ is the speed of the mean flow. The governing equations are non-dimensionalised based on the aerofoil chord length $L_{c}$ for length scales, the ambient speed of sound $a_{\infty}$ for velocities, $L_{c} / a_{\infty}$ for time scales and $\rho_{\infty} a_{\infty}^{2}$ for pressure. Temperature, density and dynamic viscosity are normalised by their respective ambient values: $T_{\infty}, \rho_{\infty}$ and $\mu_{\infty}$.

The governing equations are numerically solved using high-order accurate numerical methods on a structured multiblock grid system. The flux derivatives calculation is based on eighth-order pentadiagonal compact finite difference schemes with five-point stencil\$30. Numerical stability is preserved by a sixth-order pentadiagonal compact filter for which the cutoff wavenumber (normalised by the grid spacing) is set to $0.7 \pi$. An adaptive nonlinear artificial dissipation model is implemented to enhance the numerical stability near the shock wave avoiding unnecessary damping on the smooth linear acoustic waves 32 . Explicit time advancing of the numerical solution is carried out by using the classical fourth-order Runge-Kutta scheme with the CFL number of 0.95. Characteristic non reflecting boundary conditions $\sqrt{33}$ are coupled with the sponges in the far field boundaries. Periodic conditions are used across the spanwise boundary planes and characteristic interface conditions are imposed at the boundaries that separate two blocks. Noslip wall boundary conditions are implemented on the aerofoil surface 34 . The code is fully parallelised via domain decomposition and message passing interface (MPI) libraries, where the distributed solution of the pentadiagonal matrix systems is achieved with a quasi-disjoint approach 35 . 


\begin{tabular}{cc}
\hline & Abbreviation \\
\hline Wavy Leading Edge & WLE \\
Laminar-Turbulent & L-T \\
Leading Edge & LE \\
Trailing Edge Device & TED \\
Vortex Generator & VG \\
Shock Wave Boundary Layer Interaction & SBLI \\
Shock Control Bump & SCB \\
Straight Leading Edge & SLE \\
Direct Numerical Simulation & DNS \\
Large Eddy Simulation & LES \\
Angle of Attck & AoA \\
Boundary layer & BL \\
Laminar Separation Bubble & LSB \\
Implicit Large Eddy Simulation & ILES \\
Trailing Edge & TE \\
Inverse Distance Weighting & IDW \\
Streamwise Vortex & SV \\
\hline
\end{tabular}

TABLE I: List of the abbreviations used in the paper.

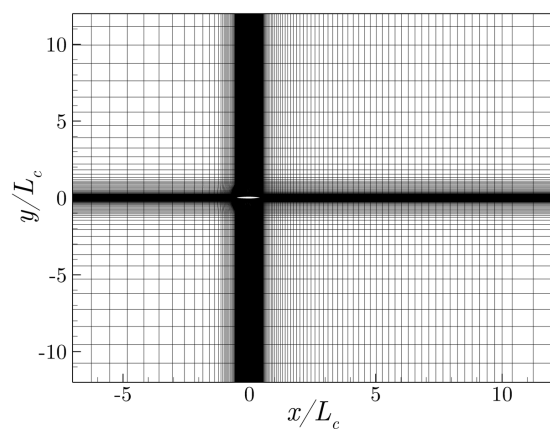

(a)

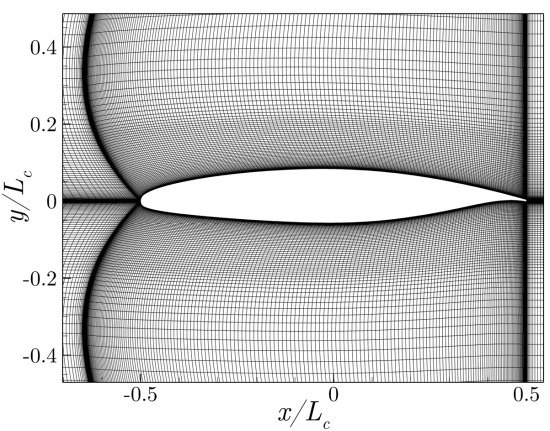

(b)

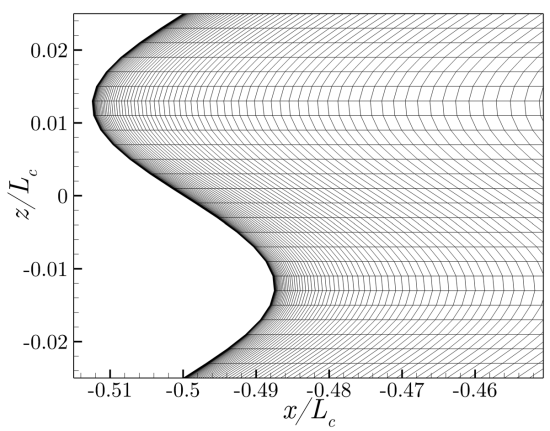

(c)

FIG. 1: Computational grid. Full domain (a), 16\% of points shown. Close up near the aerofoil (b), $25 \%$ of points shown. Top view of wavy leading edge (c), $50 \%$ of points shown.

\section{A. Geometry and discretisation of the problem}

The computational domain is shown in Figure 1a A structured multi-block grid is used based on a H-topology, stretched in both streamwise and vertical directions. A very fine grid spacing is imposed close to blocks boundaries to avoid loss of accuracy due to boundary compact schemes (Figure 1b). Figure 1a shows that the grid cells are clustered vertically one chord above and below the aerofoil and the grid becomes even finer in the region $y= \pm 0.2 L_{c}$ (Figure 1b) in order to capture properly the interaction between the shock wave and the boundary layer.

The aerofoil geometry is given by

$$
\left\{\begin{array}{l}
x_{B}(z)=k_{w}(z)\left(x_{A}-x_{\mathrm{TE}}\right)+x_{\mathrm{TE}} \\
y_{B}(z)=k_{w}(z)\left(y_{A}-y_{\mathrm{TE}}\right)+y_{\mathrm{TE}}
\end{array}\right.
$$

where

$$
k_{w}=1-\frac{h_{L E}}{L_{c}} \sin \left[\frac{2 \pi\left(z-z_{0}\right)}{\lambda_{L E}}\right],
$$

where $\left(x_{A}, y_{A}\right)$ represents the coordinates of the original (unmodified) aerofoil section, and $\left(x_{B}, y_{B}\right)$ the corresponding coordinates of the modified aerofoil section varying with the spanwise coordinate. The spanwise domain size is denoted by $L_{z}$ and it is set to $0.05 L_{c}$. In this work, $h_{L E}=0.0125 L_{c}$ and $\lambda_{L E}=0.05 L_{c}$ (Figure 1c), thus only one wavelength is considered for now. The amplitude is chosen consistent with previous large eddy simulations in low speed flows 20136 . The amplitude has to be limited using a laminar supercritical aerofoil in order to not loose the benefits of the laminar 
boundary layer. The aspect ratio of the wavy geometry $\left(2 h_{L E} / \lambda_{L E}\right)$ is 0.5 . Figure $1 \mathrm{c}$ shows that the waviness at the leading edge stretches the grid cells, thus the computational cost increases. In particular, it is observed that an aspect ratio equal to one doubles the computational cost of the simulation compared with the baseline case. The aerofoil is placed at the centre of the domain with the origin at the mid-chord location. The domain of investigation comprises $x \in[-7,12]$ in the streamwise direction, $y \in[-12,12]$ in the vertical direction and $z \in\left[-L_{z} / 2, L_{z} / 2\right]$ in the spanwise direction.

The selected grid consists of $1.1 \times 10^{6}$ cells on each $x y$-plane with 1430 cells in streamwise direction and 800 cells in vertical direction. For each wavelength of the WLE, 50 cells are used as done in a previous ILES study 20. Since no turbulence model is included, a sufficiently high level of near-wall grid resolution is maintained in order to properly resolve the boundary layers throughout the surface. Figure 2a shows the distribution of mean wall variables over the SLE aerofoil. The wall variables constraints are not applicable to laminar boundary layers and this is an advantage of laminar aerofoils. In this case, the flow is always laminar up to $x=-0.15$, thus the wall variables are checked downstream this position. In the direction normal to the aerofoil, the highest value of the distribution $\left(\Delta y_{\max }^{+}=0.7\right)$ is below one. In the streamwise direction $\left(\Delta x_{m a x}^{+}=36\right)$, it is between the DNS and the LES range $\mathrm{e}^{37}$, while in the spanwise direction $\left(\Delta z_{\max }^{+}=18\right)$, it is in the low LES range. A finer spanwise grid (75 cells) is considered, where the spanwise wall variable is between the DNS and the LES range. The pressure coefficient distribution for the selected grid G1 and the finer grid G2 are shown in Figure 2b It is compared the distribution at $\alpha_{g}=2.30^{\circ}$ and in order to obtain a mean turbulence, a mean pressure coefficient is evaluated because the angle of attack is slowly changing. The instantaneous lift coefficient for G1 grid is $C_{L}=0.57$, while for G2 grid it is $C_{L}=0.55$. The selected grid G1 consists of $57.2 \times 10^{6}$ total cells and due to the imposed Reynolds number the time step has an order of magnitude of $10^{-5}$. The computational cost is 2 wall-clock hours per time unit using 2,592 processors cores in the UK national supercomputer ARCHER, which brings to a max of 26,500 points per processor.

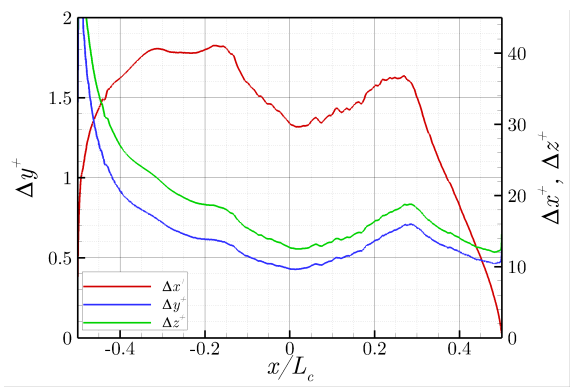

(a)

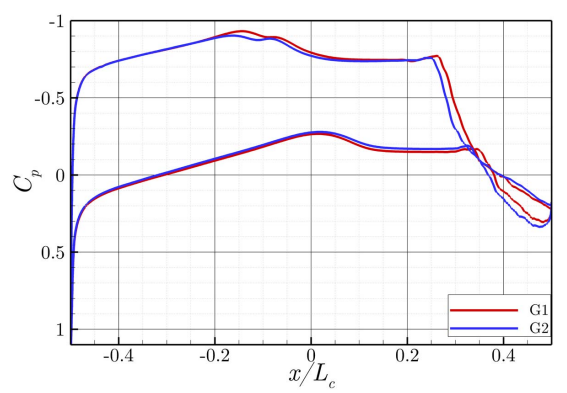

(b)

FIG. 2: Wall variables mean distribution during the heaving period along the chord of SLE aerofoil (a) and mean pressure coefficient distribution for G1 and G2 meshes at $\alpha_{g}=2.30^{\circ}$ b.

\section{B. Heaving motion}

Since this is the first work about WLE performance in transonic flows, the choice is to conduct an analysis varying the angle of attack. The high computational cost of the simulation justifies the choice to perform a slow heaving motion to analyse the aerodynamic performance as a function of the incidence angle.

The upstream flow is given by $\left(u_{\infty}, v_{\infty}, w_{\infty}\right)=U_{\infty}(\cos \alpha, \sin \alpha, 0)$ with the aerofoil mean chord parallel to the $x$-axis. In Eq. (1), $\hat{u}_{j}=\{\hat{u}, \hat{v}, \hat{w}\}$ is the velocity of the moving frame relative to the mean flow velocity, in order to describe the slow heaving motion of the aerofoil, of which the derivation is found in Hoffmann and Chiang ${ }^{38}$. The current slow periodic heaving motion is given by

$$
\hat{v}(t)=\frac{\epsilon U_{\infty}}{2}\left[\cos \left(2 \pi \frac{t-t_{0}}{T}\right)-1\right] \quad \text { with } \quad \hat{u}(t)=\hat{w}(t)=0,
$$

where $\epsilon$ is chosen to set the maximum geometric angle of attack to $\alpha_{g}=7^{\circ}$, and the period of the heaving motion is $T a_{\infty} / L_{c}=50$ which corresponds to a Strouhal number of 0.0285 . The heaving motion is initiated from $t_{0} a_{\infty} / L_{c}=5$ when the mean flow has settled down after an initial condition.

Figure 3 illustrates the AoA profile. The extremes are chosen in order to obtain a wide range within the limits of the transonic regime. While there are extensive studies of $\mathrm{V} 2 \mathrm{C}$ aerofoil at the upper incidence angle ${ }^{39}$, the lower one has never been considered for this aerofoil. 


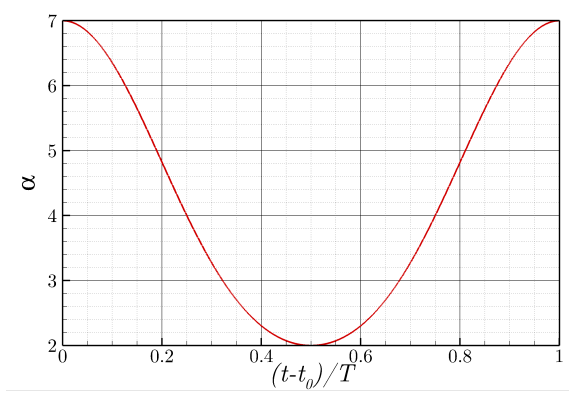

FIG. 3: Angle of attack profile used for slow heaving motion simulations.

\section{AERODYNAMIC PERFORMANCE IN QUASI-LINEAR MOTION}

The main focus of the paper is on the quasi-linear part of the heaving motion, but an overview of the full heaving motion is given in Appendix A. The aerodynamic performance of the baseline and the modified aerofoils is analysed in details both in streamise and spanwise directions. In this study low incidence angles are considered in the range $\alpha_{g} \leq 3.5^{\circ}$, moderate angles in the range $3.5^{\circ}<\alpha_{g}<5.0^{\circ}$ and high angles in the range $\alpha_{g} \geq 5.0^{\circ}$. Figure 4 illustrates the performance in quasi-linear range, showing the lift and drag coefficients variation with the angle of attack. The main outcome is that the modified aerofoil decreases the drag at low and moderate incidence angles, the result is an increased efficiency. Increasing the angle of attack, the drag coefficient of the two aerofoils tends to the same value, the same trend is observed for the efficiency. This suggests that the aerodynamic characteristics of WLEs in transonic flows are different from the ones in low-speed flows. A static analysis is required at higher angles of attack because of low frequency unsteadiness.

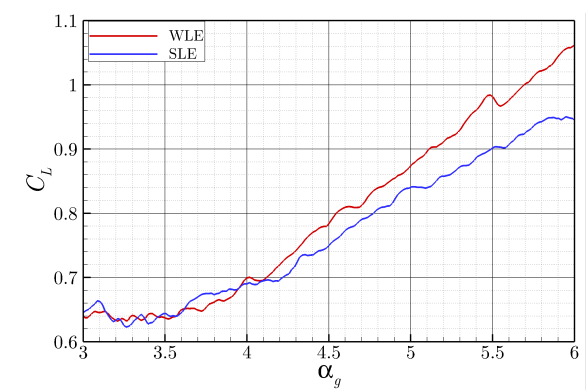

(a)

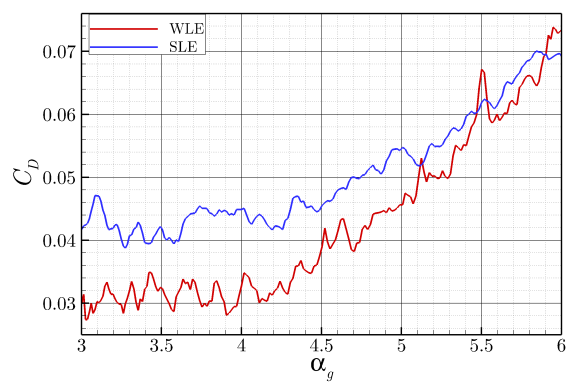

(b)

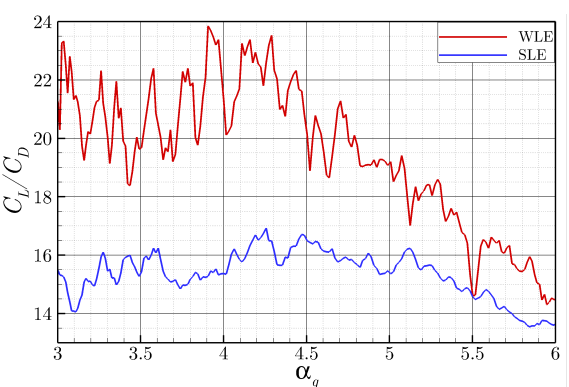

(c)

FIG. 4: Lift (a) and drag (b) coefficients and efficiency (c) varying the angle of attack during the quasi-linear part of the heaving motion.

\section{A. Streamwise aerodynamic performance details}

The quasi-linear part of the heaving motion is analysed in detail trying to understand the flow behaviour and comparing the performance between the two aerofoils. The performance is evaluated considering the piecewise distributions of lift and drag increase/decrease along the chord $\left(d\left(\Delta C_{L}\right) / d x\right.$ and $\left.d\left(\Delta C_{D}\right) / d x\right)$ between SLE and WLE cases.

Figure 5 represents the pressure gradient magnitude contour highlighting the supersonic region with a solid red line at different incidence angles of the quasi-linear motion for the straight (Figure $5 \mathrm{k}-\mathrm{c}$ ) and the wavy (Figure $5 \mathrm{~d}-\mathrm{f}$ ) aerofoils. At low and moderate angles, pressure waves are generated at the trailing edge and they gather downstream the supersonic region generating a weak shock wave on the upper surface (Figure $5 \mathrm{a}, \mathrm{d}$ ). The shock wave moves upstream decreasing its strength until it reaches the leading edge, then it separates from the LE and moves upstream as a free shock. This behaviour can be observed up to $\alpha_{g}=5.72^{\circ}$ on the baseline aerofoil (Figure $5 \mathrm{a}-\mathrm{c}$ ), while at that angle the WLE case shows a $\lambda$-shape shock wave (Figure 5f). Figure 5b-i represents the pressure coefficient distribution over the main sections of the two aerofoils at different AoA. The general trend of the pressure coefficient over the upper surface of the laminar supercritical aerofoil is characterised by a first plateau with a laminar boundary 
layer, followed by a bump which defines the laminar separation and a second plateau before the laminar-turbulent transition and the pressure coefficient increase 40 . The pressure distribution at the main sections of the two aerofoils allows to observe some differences in the flow behaviour. First of all, the L-T transition point is moved upstream in the modified aerofoil over a wide range of angles of attack. Table III shows that increasing the incidence angle, the transition point of the wavy aerofoil tends to the one of the baseline aerofoil. Moreover, downstream the transition, the pressure coefficient increase is steeper over the modified aerofoil and this has an influence on the lift performance. The laminar separation point is moved slightly upstream at low incidence (Figure $5 \mathrm{~s}$ ), while it is moved downstream at moderate and high incidence angles (Figure $5 \mathrm{~h}, \mathrm{i}$ ). The lift and drag coefficients for each aerofoil at the angles of attack considered are reported in Table II. The WLE lift coefficient is lower only at high angles, while the WLE drag tends to be lower up to $\alpha_{g}=5.72^{\circ}$, then it is close to the straight one. The lift coefficient at $\alpha_{g}=3.08^{\circ}$ is increased upstream the L-T transition point over the modified aerofoil even if the leading edge flow itself gives a negative contribution to the lift (Figure 51). At higher angles, the lift increase is concentrated between the SLE laminar separation and the WLE transition point (Figure $5 \mathrm{~m}, \mathrm{n}$ ). The drag coefficient at low angles of attack is decreased over the modified aerofoil at the LE. The drag gain obtained close to the leading edge decreases when the incidence angle is increased (Figure 5p-q). Downstream the L-T transition point, the drag is decreased over the wavy aerofoil and also this behaviour tends to decrease, increasing the incidence angle but it becomes predominant at higher angles. Table IV]gives further details integrating in two steps the piecewise distribution of lift and drag along the chord, shown in Figure 5]-q. The first part goes from the LE up to the WLE laminar-turbulent transition point and the second part goes from the WLE transition point to the TE. In this way, the effect of the main pressure distribution features are quantified. It is observed that the lift increase with the incidence angle is related to the increased positive contribution upstream the L-T transition point and the decreased negative contribution downstream the transition point. The drag coefficient is decreased upstream the transition point at $\alpha_{g}=3.08^{\circ}$, while it is increased at $\alpha_{g}=5.72^{\circ}$. Downstream the transition point, the drag is always decreased by the modified aerofoil but the effect is reduced increasing the angle of attack.

The main outcome of the chordwise performance analysis is that the modified leading edge has a direct positive impact on the performance at low and moderate angles, but it also has an effect on the shock wave-boundary layer interaction region and on the position of the laminar-turbulent transition point. This effect seems to have a positive impact on the performance over a wide range of angles of attack. The next section will try to analyse the flow field close to the leading edge in order to understand both direct and indirect effects.

\begin{tabular}{ccccc}
\hline$\alpha_{g}[\mathrm{deg}]$ & $C_{L_{W L E}}$ & $C_{L_{S L E}}$ & $C_{D_{W L E}}$ & $C_{D_{S L E}}$ \\
\hline 3.08 & 0.646 & 0.663 & 0.029 & 0.047 \\
4.15 & 0.709 & 0.694 & 0.030 & 0.043 \\
5.72 & 1.004 & 0.929 & 0.062 & 0.066 \\
\hline
\end{tabular}

TABLE II: Lift and drag coefficients for SLE and WLE aerofoils during quasi-linear part of heaving motion.

\begin{tabular}{cccc}
\hline & $3.08^{\circ}$ & $4.15^{\circ}$ & $5.72^{\circ}$ \\
\hline$x_{t_{S L E}}$ & 0.28 & 0.23 & 0.19 \\
$x_{t_{W L E}}$ & 0.24 & 0.19 & 0.18 \\
\hline
\end{tabular}

TABLE III: Laminar-turbulent transition point over the SLE and WLE aerofoils during quasi-linear part of heaving motion.

\begin{tabular}{ccccccc}
\hline$\alpha_{g}[\mathrm{deg}]$ & $\Delta C_{L_{\text {front }}}$ & $\Delta C_{L_{\text {rear }}}$ & $\Delta C_{L}$ & $\Delta C_{D_{\text {front }}}$ & $\Delta C_{D_{\text {rear }}}$ & $\Delta C_{D}$ \\
\hline 3.08 & 0.0246 & -0.0348 & -0.0103 & -0.00375 & -0.00773 & -0.01148 \\
4.15 & 0.0255 & -0.0112 & 0.0143 & -0.000762 & -0.00649 & -0.00725 \\
5.72 & 0.0798 & -0.0037 & 0.0760 & 0.00829 & -0.00579 & 0.00251 \\
\hline
\end{tabular}

TABLE IV: Difference in lift and drag forces between WLE and SLE cases during the quasi-linear part of heaving motion $\left(\Delta C_{L}=C_{L_{W L E}}-C_{L_{S L E}}\right.$ and $\left.\Delta C_{D}=C_{D_{W L E}}-C_{D_{S L E}}\right)$, over two sections of the aerofoil chord (front and rear). The front section is from the LE to the WLE laminar-turbulent transition point and the rear section is from the WLE laminar-turbulent transition point to the TE. Viscous contributions are neglected. 


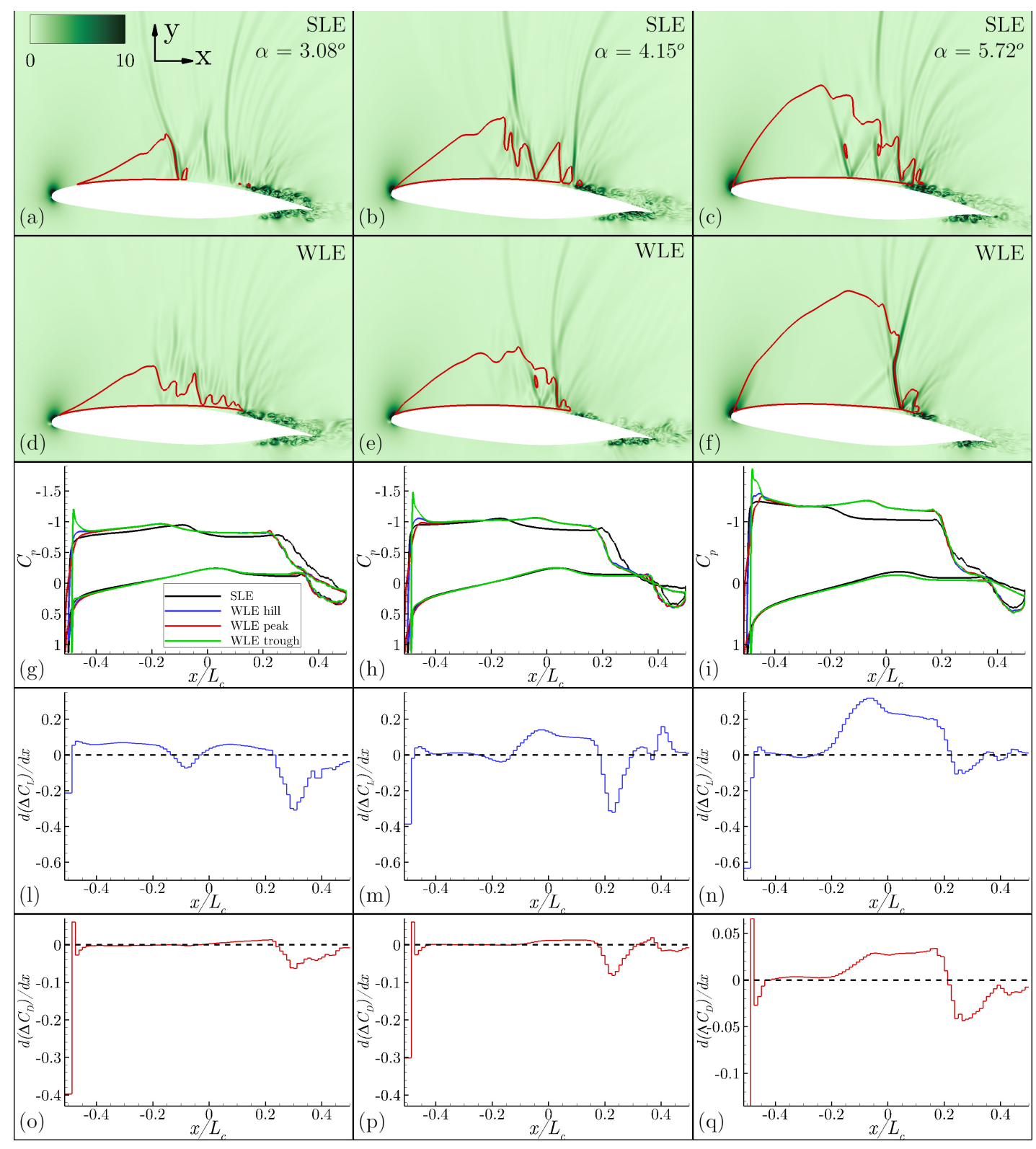

FIG. 5: Aerodynamic performance analysis during the quasi-linear part of heaving motion. Pressure gradient magnitude of SLE (a-c) and WLE hill (d-f) are shown at different angles of attack $\left(\alpha_{g}=3.08^{\circ}, 4.15^{\circ}, 5.72^{\circ}\right)$. The red solid line delimits the supersonic region. Mean pressure coefficient distribution at WLE peak, trough and hill and over SLE (g-i) are compared at different angles of attack. Relative changes in aerodynamic forces between SLE and WLE cases in terms of piecewise distribution of lift (l-n) and drag (o-q) along the chord calculated from wall pressure $\left(d\left(\Delta C_{L}\right) / d x\right.$ and $\left.d\left(\Delta C_{D}\right) / d x\right)$. The relative difference between SLE and WLE cases are denoted by

$$
\Delta\{.\}=\{.\}_{W L E}-\{\cdot\}_{S L E} .
$$

\section{B. Spanwise aerodynamic performance details}

The wavy geometry introduces three dimensionality in the flow at the leading edge and this has an effect over the whole aerofoil. For this reason, the spanwise performance is also analysed in order to define the most significant sections in terms of aerodynamic efficiency.

The spanwise performance of the modified aerofoil is analysed in Figure 6 at a specific angle of attack $\left(\alpha_{g}=4.82^{\circ}\right)$ but the behaviour is consistent at the other angles. The lift coefficient (Figure 6a) shows two different maxima at the peak and trough sections and the minimum close to the hill section. The mean lift coefficient is $C_{L_{\text {mean }}}=0.857$. The lowest value is obtained at the hill section for a geometric reason, the derivative of the surface with respect to 


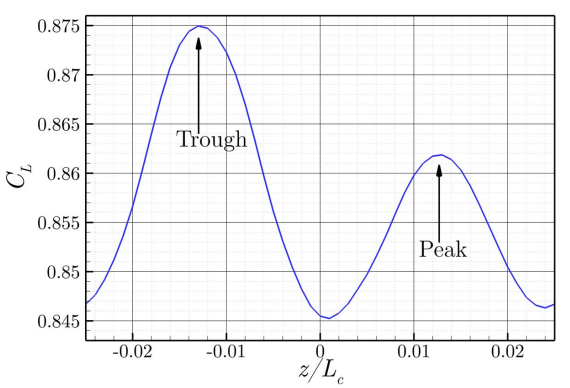

(a)

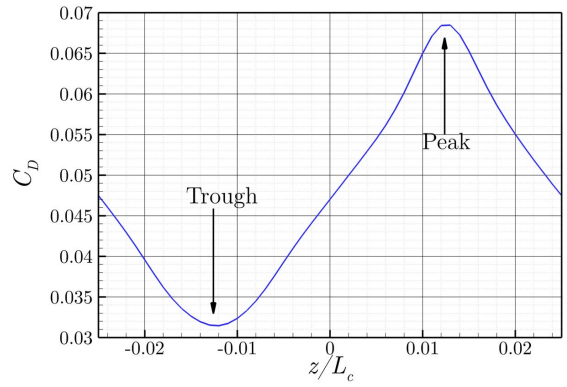

(b)

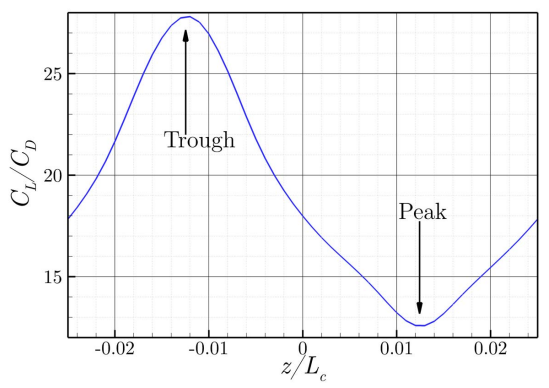

(c)

FIG. 6: Lift (a), drag (b) and efficiency (c) spanwise distribution over the wavy aerofoil at $\alpha_{g}=4.82^{\circ}$.

the $z$ direction has the highest value so the force has the largest spanwise component. The trough generates a lift higher than the peak because of the pressure distribution close to the leading edge. The result is quite different for the drag coefficient (Figure 6b). The highest value is obtained at the peak and the lowest at the trough. The result is consistent for both pressure and skin friction drag even if the first one is almost one order of magnitude larger. The mean drag coefficient is $C_{D_{\text {mean }}}=0.0478$, close to the hill section value. This spanwise distribution can be explained again considering the pressure distribution close to the LE. The peak section is the one where there is the most gradual pressure decrease between the stagnation point and the pressure coefficient peak. This extended high pressure region increases the drag. The opposite behaviour is observed at the trough, the pressure coefficient changes suddenly from the highest to the lowest values of the pressure coefficient. Overall, the efficiency is 1.5 of the mean one at the trough and 0.72 at the peak (Figure 6r).

\section{LEADING EDGE FLOW DETAILS IN QUASI-LINEAR MOTION}

The previous section has analysed the performance of the modified aerofoil in detail and compared it with the baseline one. The objective of this section is to analyse the leading edge flow field and its influence on the downstream evolution of the flow, trying to compare the main flow characteristics with the ones at low speed. The first part investigates the leading edge pressure distribution along the span and compares it with the straight counterpart. Then the laminar boundary layer downstream the LE region is evaluated in order to quantify the spanwise variation of BL thickness over wavy aerofoil. The second part examines the evolution of streamwise vortices from the LE to the L-T transition point and the spanwise velocity upstream and downstream the LE (channeling effect).

\section{A. Leading edge acceleration and boundary layer evolution}

The chordwise variation of aerodynamic forces has shown that the drag decrease over the modified aerofoil is related to the leading edge shape and the upstream movement of the transition point. The first effect is dominant at low and moderate angles of attack. Figure 5 shows that the peak region has a positive effect in decreasing the drag, while the trough region has a negative effect. In particular, a drag drop is obtained in the first chunk $x \in\left[-0.5125 L_{c} ;-0.4875 L_{c}\right]$, while a drag increase is observed in the second one $x \in\left[-0.4875 L_{c} ;-0.4750 L_{c}\right]$. Increasing the angle of attack, the favourable effect of the first chunk decreases while the adverse effect of the second one increases and overall the leading edge is not beneficial. The favourable behaviour observed in the first chunk can be explained analysing the spanwise distribution of the maximum pressure coefficient (Figure 7) because a high pressure coefficient at the leading edge tends to increase the drag. The maximum pressure coefficient is close to the straight one at the trough and peak sections but it is lower at the other sections. In particular, the maximum pressure coefficient decreases abruptly around the peak, while it decreases gradually around the trough. The spanwise distribution of the streamwise location of the maximum pressure coefficient is not shown but it follows the shape of the wavy geometry. It is located on the lower surface close to the leading edge and it moves further away from the leading edge increasing the angle of attack. The outcome of this pressure distribution is that the first chunk, which takes into account the peak and the hill effects, brings to a drag reduction. The reduction in the first chunk decreases with the incidence angle increase because the maximum pressure coefficient drop decreases at higher angles. On the contrary, in the second chunk the high pressure coefficient in the trough region penalises the wavy aerofoil performance and this effect grows increasing the incidence angle because the high pressure region moves downstream and an higher pressure area is included in 
the second chunk.

The flow field at the leading edge over the two aerofoils is illustrated in Figure 8, where the contour of streamwise velocity is represented together with the sonic line. The first observation is related to the spanwise boundary layer thickness over the wavy aerofoil. At the trough section a reverse flow region develops inside the boundary layer and becomes larger increasing the angle of attack. The effect of this reverse flow region is boundary layer thickening. The effect of the reverse flow seems to be limited to the trough area because the boundary layer is thinner at the hill and peak sections and it seems to be comparable with the boundary layer over the baseline aerofoil.

Figure 9 shows the details of the LE bubble around the trough region at $\alpha_{g}=6.00^{\circ}$. It is shown only one specific condition but the results are consistent at the other incidence angles with the bubble in the trough region growing with the angle of attack increase. The bubble canopy (tangential velocity equal to zero) and the streamlines on both side and top views allow to understand the details of the flow behaviour in the reverse flow region. Two foci are displayed clearly in the top view and they are consistent with previous studies of WLE aerofoils at low speed 20 . The side view showing the velocity vectors allows to analyse the boundary layer details in the bubble. Downstream the laminar separation, the BL in the reverse flow is qualitatively similar to the BL in a channel, the flow is zero at the wall, then it grows in magnitude to a maximum and then it goes to zero again before becoming positive. Further downstream the BL changes, it is negative close to the wall but with a small amplitude and it is almost constant moving away from the wall. In the upper part of the reverse flow region, the magnitude starts growing reaching a negative maximum before going to zero and becoming positive. At the end of the reverse flow region, the BL shape is evolving again. Close to the wall the velocity is negative in a very limited region, then it becomes positive reaching a maximum before showing a strong deceleration and becoming slighty negative again. Finally, outside the reverse flow region it becomes positive and it quickly grows to supersonic conditions.

A reverse flow region has a positive effect on the skin friction drag. However, in this case the skin friction drag is about one order of magnitude lower than the pressure drag mainly because of the aerofoil shape, a laminar supercritical aerofoil is designed to decrease the skin friction drag exploiting the characteristics of a laminar boundary layer. Moreover, the reverse flow region has a limited streamwise and spanwise extension (Figure 9), this means that overall the effect of the reverse flow region on the local drag at the LE is a secondary effect.

The second relevant point about the flow field at the leading edge is related to the flow acceleration and the supersonic region. Figure 8 shows clearly that the supersonic region is very close to the LE at the trough section, while it moves further downstream at the hill and peak. A possible explanation for the flow behaviour at the trough section will be given in the next section analysing the channeling phenomenon. Table V gives quantitative information about the position of the sonic point closest to the wall and its distance from the local leading edge point. Obviously, the sonic point tends to move upstream increasing the angle of attack, but the range of variation increases moving from the trough to the peak. The consequence is that at low angles of attack the supersonic region starts quite far from the leading edge over the baseline aerofoil, while it starts close to the leading edge at the trough and this has an effect on the other sections where the sonic point is more upstream than the straight counterpart. On the contrary, when the sonic point moves upstream $x=-0.4875$ (trough LE) over the baseline aerofoil, the sonic point is more downstream at all the sections of the wavy aerofoil because of the trough. The flow behaviour in this region has an impact on the drag performance of the modified aerofoil, where it allows to decrease the drag at low and moderate angles of attack, while it plays a minor role at high angles.

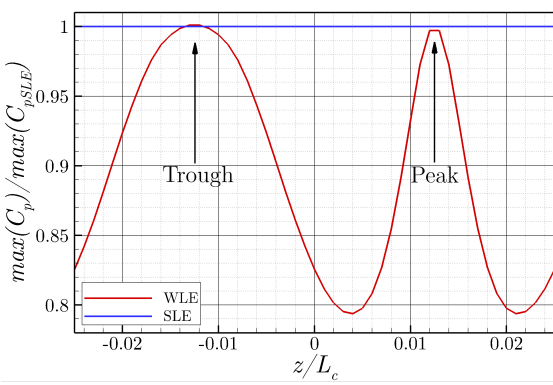

(a)

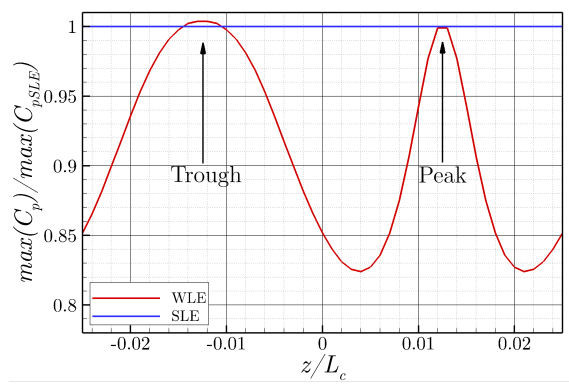

(b)

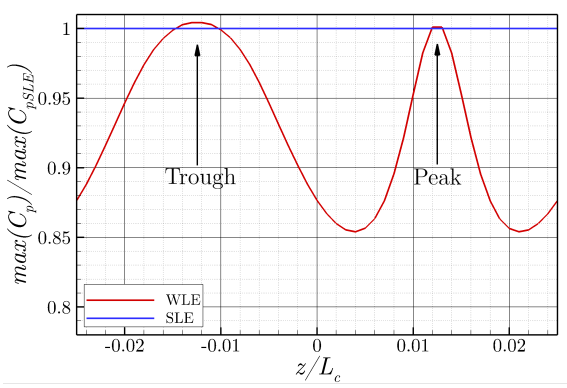

(c)

FIG. 7: Spanwise maximum pressure coefficient normalised by the baseline aerofoil one at $\alpha_{g}=3.00^{\circ}$ (a), $\alpha_{g}=4.82^{\circ}$ (b) and $\alpha_{g}=6.00^{\circ}(\mathrm{c})$.

Figure 8 shows a large spanwise variation of the boundary layer thickness over the modified aerofoil. The objective is to quantify the thickness and its evolution downstream. Since the standard definitions of displacement thickness and boundary layer thickness can not be applied to an aerofoil due to the wall curvature, a different approach is 


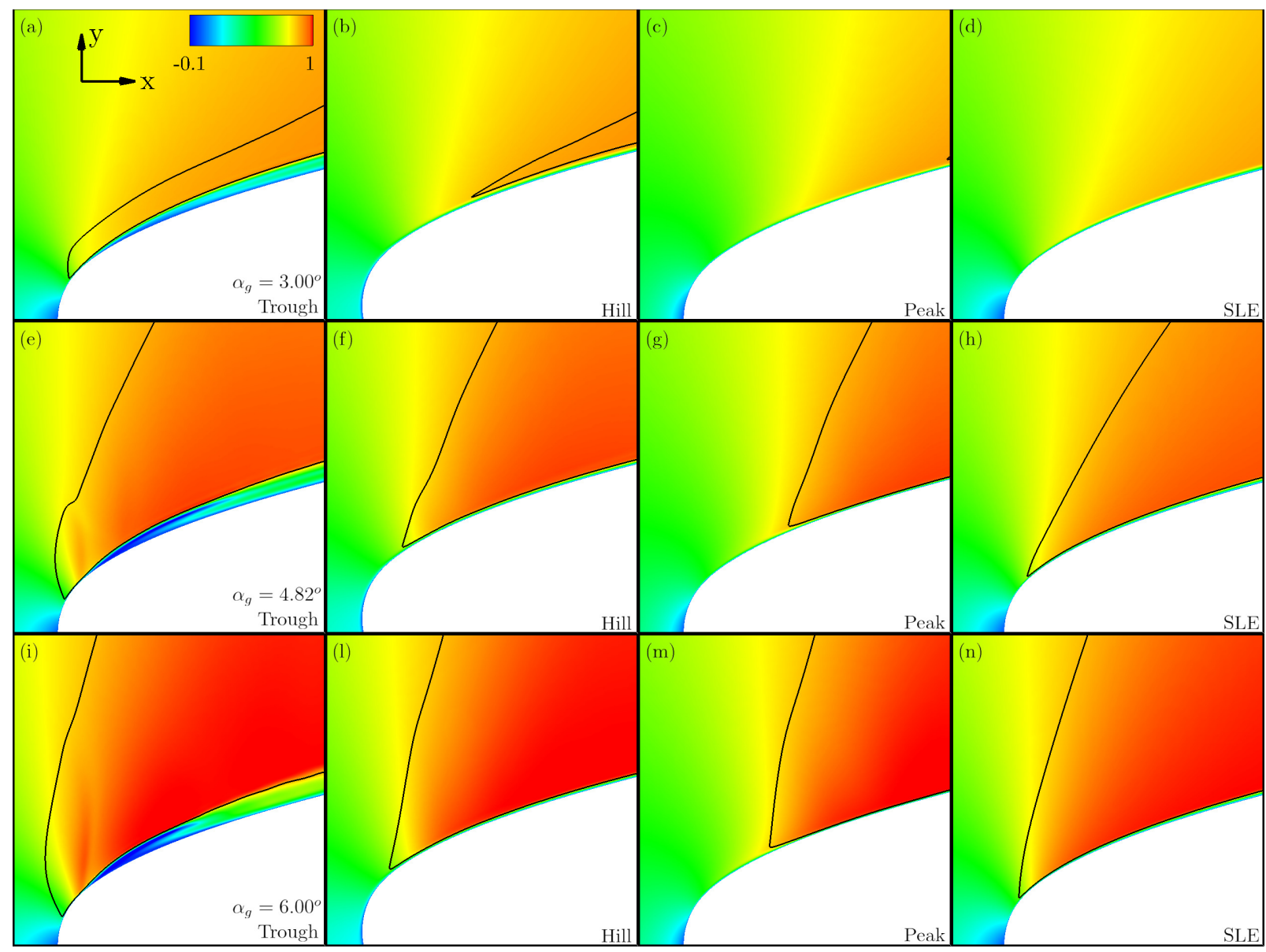

FIG. 8: Streamwise velocity at trough $(\mathrm{a}, \mathrm{e}, \mathrm{i})$, hill $(\mathrm{b}, \mathrm{f}, \mathrm{l})$ and peak $(\mathrm{c}, \mathrm{g}, \mathrm{m})$ sections of WLE aerofoil and over the baseline aerofoil $(\mathrm{d}, \mathrm{h}, \mathrm{n})$ for $\alpha_{g}=3.00^{\circ}(\mathrm{a}-\mathrm{d}), \alpha_{g}=4.82^{\circ}(\mathrm{e}-\mathrm{h})$ and $\alpha_{g}=6.00^{\circ}(\mathrm{i}-\mathrm{n})$. The black line highlights the sonic line.

\begin{tabular}{ccccccccc}
\hline$\alpha_{g}[\mathrm{deg}]$ & $x_{\text {trough }} / L_{c}$ & $x_{\text {hill }} / L_{c}$ & $x_{\text {peak }} / L_{c}$ & $x_{S L E} / L_{c}$ & $\Delta x_{\text {trough }} / L_{c}$ & $\Delta x_{\text {hill }} / L_{c}$ & $\Delta x_{\text {peak }} / L_{c}$ & $\Delta x_{S L E} / L_{c}$ \\
\hline 3.00 & -0.4843 & -0.4698 & -0.4411 & -0.4019 & 0.0032 & 0.0320 & 0.0714 & 0.0981 \\
4.82 & -0.4860 & -0.4891 & -0.4839 & -0.4940 & 0.0015 & 0.0109 & 0.0286 & 0.0060 \\
6.00 & -0.4864 & -0.4927 & -0.4890 & -0.4959 & 0.0011 & 0.0073 & 0.0235 & 0.0041 \\
\hline
\end{tabular}

TABLE V: Streamwise coordinates of the most upstream position of the sonic line close to the wall and its distance from the local LE for WLE trough, hill, peak and for SLE $\left(\Delta x_{\{.\}}=x_{L E}-x_{\{.\}}\right)$.

exploited.

- First of all, the solution in the coordinates system $(x, y)$ is transformed in the system $(s, \eta)$, where $s$ is parallel to the aerofoil surface and the origin is at the leading edge, while $\eta$ is normal to the surface and the origin is at the wall. This transformation is obtained using inverse distance weighting (IDW) interpolation and an example is illustrated in Figure 10

- The result of the first step is the velocity magnitude profile of the boundary layer $(U-\eta)$ at each streamwise section. The condition to define the boundary layer edge is to have $\partial^{2} U / \partial \eta^{2}=0$ for a defined number of points. This means that the velocity magnitude is constant or it is changing linearly, thus it is for sure outside the boundary layer.

The boundary layer thickness $(\delta)$ is evaluated downstream the leading edge region, where the boundary layer is still laminar. In principle the method can be applied also to a mean turbulent boundary layer but since the aerofoils are performing a heaving motion, it is only possible to analyse the laminar one in this case. Figure 11 shows the result for the angles of attack considered in this section. At the trough section the boundary layer tends to grow close to the 


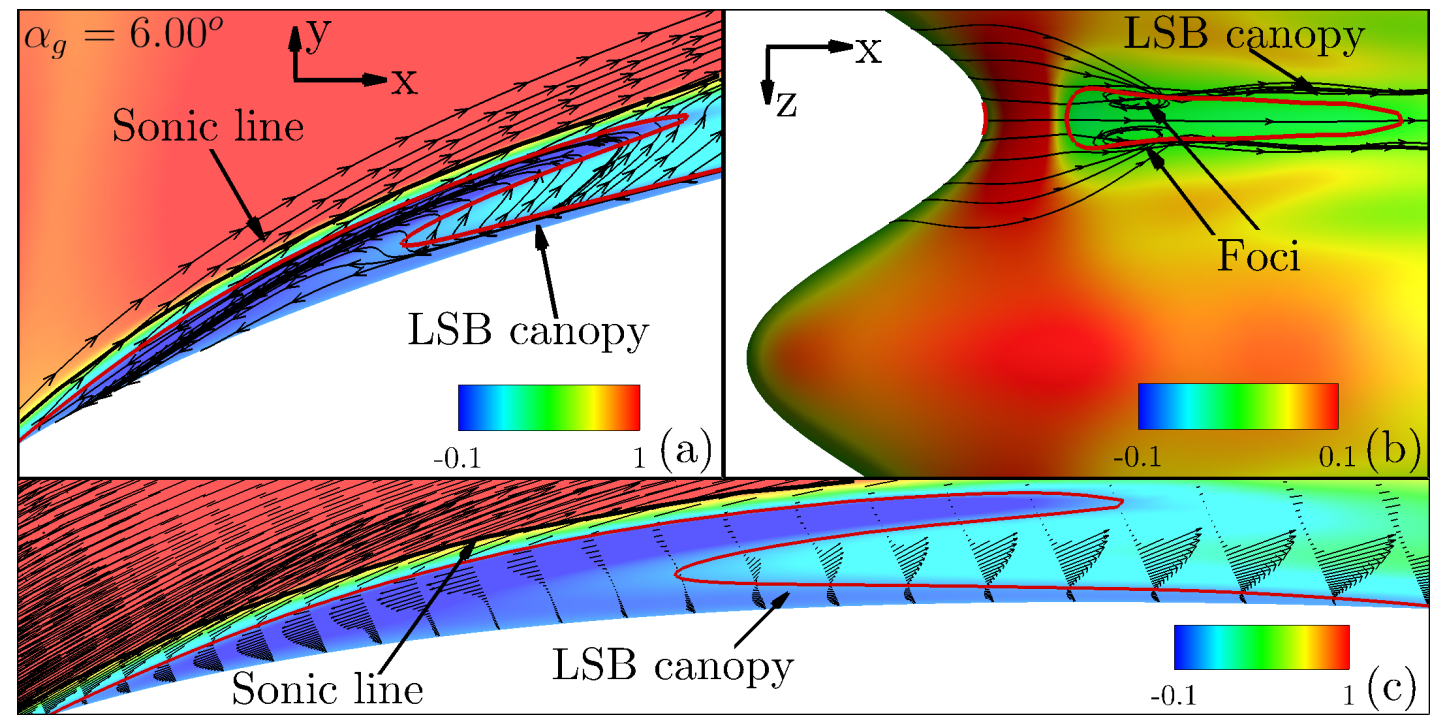

FIG. 9: Streamwise velocity contour over the aerofoil at $\alpha_{g}=6.00^{\circ}$. Side view with streamline highlighting the bubble canopy and the sonic line (a), top view close to the aerofoil surface with streamlines highlighting the bubble foci and the bubble canopy (b), side view with velocity vectors plotted every 3 points in $\xi$ direction highlighting the bubble canopy and the sonic line (c).
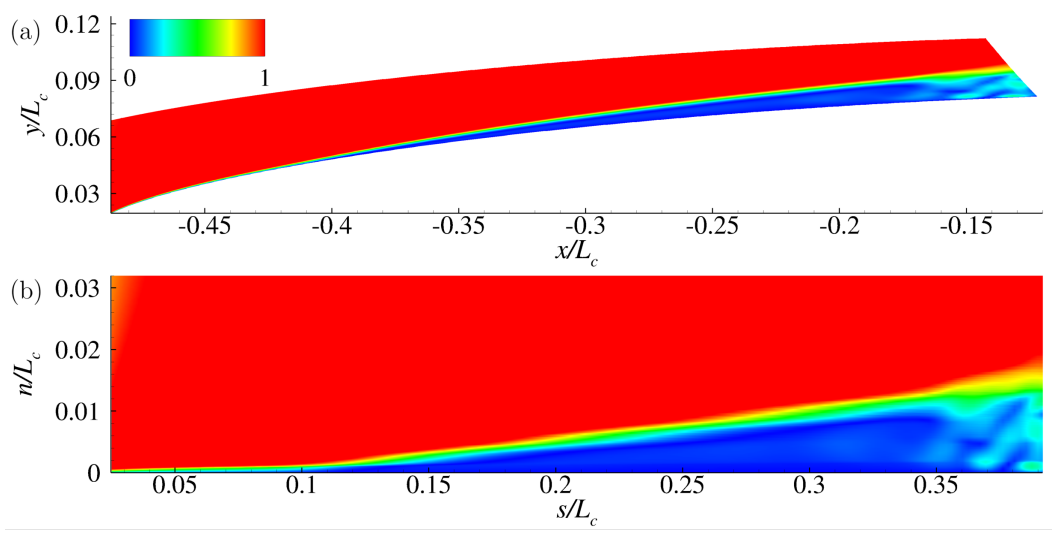

FIG. 10: Velocity magnitude close to the wall in coordinate system $(x, y)(\mathrm{a})$ and $(s, \eta)(\mathrm{b})$.

leading edge, while moving further away it becomes quite constant. A different behaviour is observed over the straight aerofoil and at the peak where the BL increases slowly but constantly. Further details are given in Table VI where the thickness at the trough, at the peak and over the SLE is evaluate at $s=0.25$ and at the different angles. The result shows that the thickness grows faster increasing the incidence angle at the trough than at the peak where it is quite constant changing the angle of attack. The growth of the BL over the SLE is similar to the trough section but the shape is quite different due to the evolution of streamwise vortices and the reverse region close to in the trough.

\begin{tabular}{cccc}
\hline$\alpha_{g}[\mathrm{deg}]$ & $\delta_{\text {trough }}$ & $\delta_{\text {peak }}$ & $\delta_{S L E}$ \\
\hline 3.00 & 0.00854 & 0.00228 & 0.00354 \\
4.82 & 0.00917 & 0.00234 & 0.00430 \\
6.00 & 0.01346 & 0.00232 & 0.00443 \\
\hline
\end{tabular}

TABLE VI: BL thickness at the trough, at the peak and over the SLE at $s=0.25$ for $\alpha_{g}=3.00^{\circ}, \alpha_{g}=4.82^{\circ}$ and $\alpha_{g}=6.00^{\circ}$. 

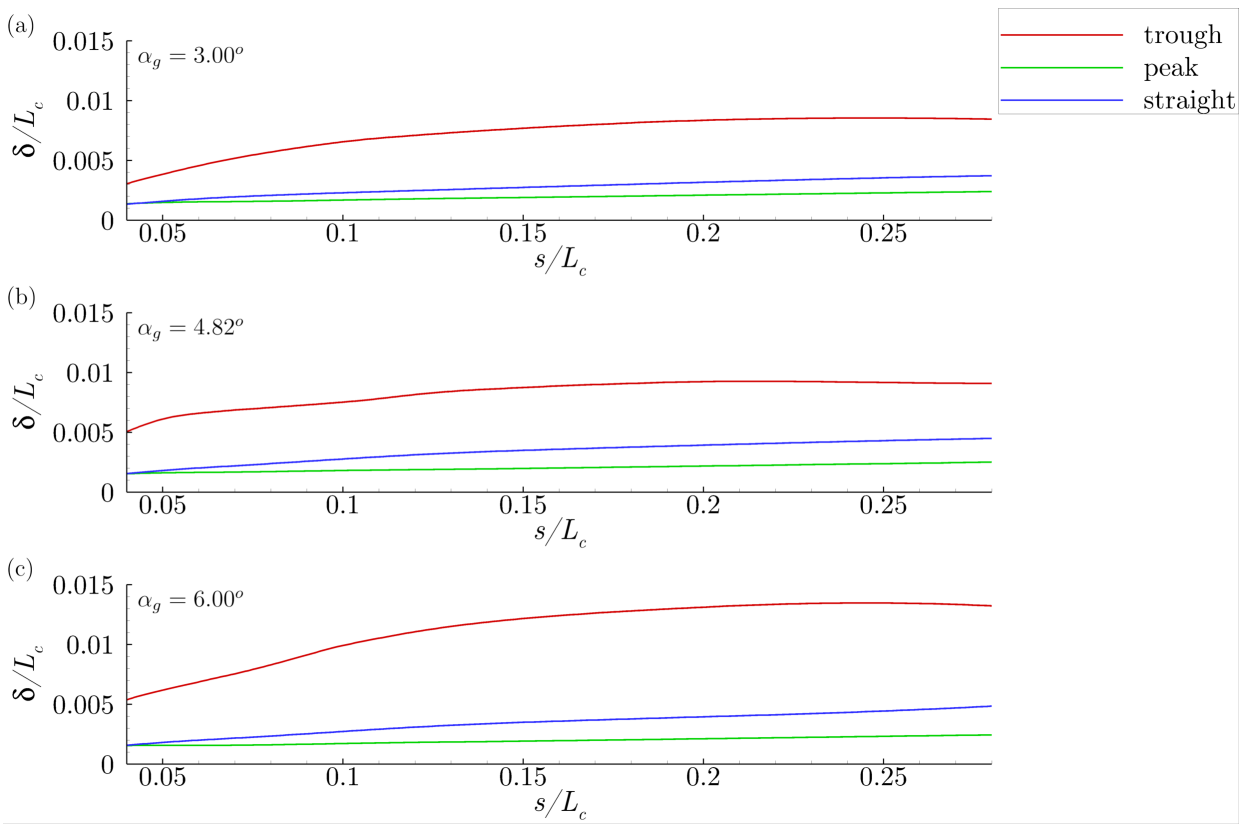

FIG. 11: Boundary layer thickness $\left(\delta / L_{c}\right)$ distribution along the curvilinear coordinate $s$ at $\alpha_{g}=3.00^{\circ}$ (a), $\alpha_{g}=4.82^{\circ}$ (b) and $\alpha_{g}=6.00^{\circ}$ (c) for WLE trough and peak and for SLE.

\section{B. Leading edge flow channeling}

The acceleration of the flow to a supersonic condition at the leading edge of the modified aerofoil was observed in Figure 8. A possible explanation of this flow behaviour is the flow channeling phenomenon illustrated in Figure 12 for $\alpha_{g}=4.82^{\circ}$. The upstream flow approaching the aerofoil is subsonic, thus it deviates before encountering the peak. This creates a spanwise velocity component towards the trough. The streamlines converge towards the trough and the subsonic flow is accelerated. In a streamwise location between trough and peak $(x=-0.5)$ a lobular pattern of the spanwise velocity can be observed. The lower lobs indicate that the flow is moving towards the trough, while on the upper side it is moving in the opposite direction, away from the trough section. The sonic line close to the wall is observed at $x=-0.4860$ (Table $\mathrm{V}$ ) and at this streamwise location the spanwise velocity component has an opposite direction, thus the streamlines diverge from the trough. The flow is supersonic downstream this location, hence the flow is still accelerated further downstream. Figure 12 shows a streamwise position downstream the sonic line at $x=-0.48$. The flow in the boundary layer is influenced by the aerofoil geometry and it moves towards the trough, while outside the boundary layer the flow channeling phenomenon is dominant and the flow diverges from the trough. The opposite spanwise velocity sign inside and outside the boundary layer generates streamwise vorticity downstream. The flow channeling phenomenon can be observed also at low speeds. If the Mach number is low, the flow accelerates upstream the leading edge during the convergence and it slows down during the divergence downstream the leading edge. In this case the flow becomes sonic before the divergence and it continues the acceleration downstream the leading edge. The flow field during the channeling phenomenon can be compared to the one inside a convergentdivergent nozzle where the flow becomes sonic at the throat. The pressure coefficient is plotted in Figure $13 \mathrm{~b}$ along the streamlines illustrated in Figure 13a. The pressure coefficient distribution at the trough recalls the one along the axis of a nozzle. The main difference is related to the presence of the body, the flow initially slows down approaching the aerofoil, but it accelerates close to the LE and it reaches sonic condition around the LE position. Downstream the leading edge, the flow close to the wall is decelerated by the presence of a reverse flow region, while moving further away from the wall (grey line) it keeps accelerating up to $x=-0.45$. Upstream the LE, the magnitude of both acceleration and deceleration increase moving closer to the wall, but overall the result is the same because the streamlines have the same pressure coefficient $C_{p}=0.4$ at $x=-0.6$ and they reach the critical pressure coefficient (sonic line) around the same position $x \simeq-0.486$. Downstream the LE, the deceleration caused by the reverse flow is stronger close to the wall due to a ramp effect related to the steep boundary layer thickening. Figure $13 \mathrm{c}$ shows the convergent-divergent shape of the flow. The throat location and the position of the sonic line are not exactly in the same position $\left(x_{M=1}-x_{t}=0.005\right)$. In this short region the spanwise pressure gradient $(\partial p / \partial z)$ slows down the flow, but the dominant streamwise pressure gradient $(\partial p / \partial x)$ speeds it up to sonic condition. Decreasing the upstream flow velocity, the distance between the throat and the sonic line increases because the sonic line moves downstream 


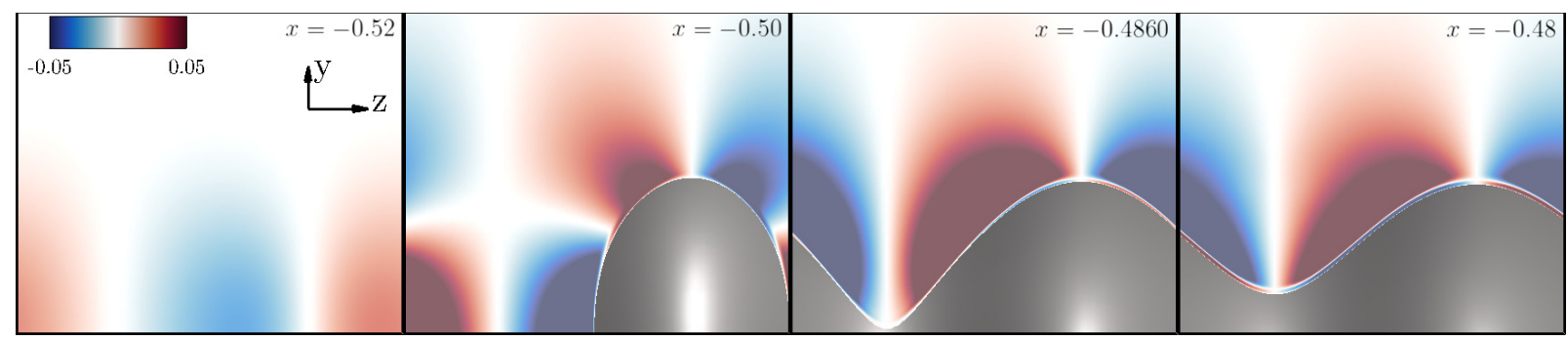

FIG. 12: Contour plots of spanwise velocity at four streamwise positions from $x=-0.52$ to $x=-0.48$ for the wavy aerofoil at $\alpha_{g}=4.82^{\circ}$.

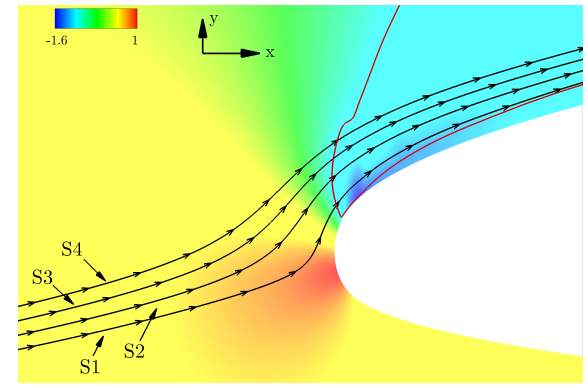

(a)

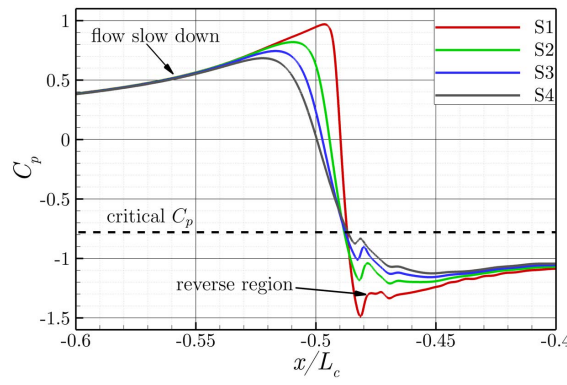

(b)

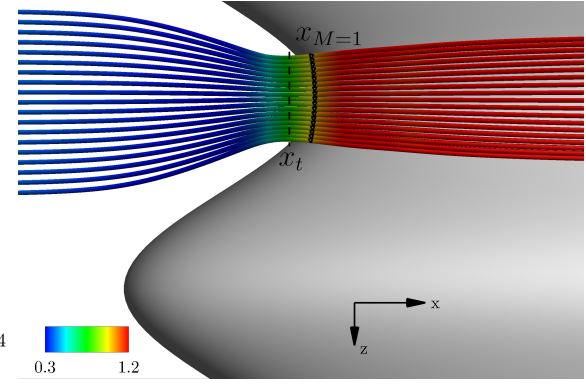

(c)

FIG. 13: Channeling phenomenon details. Pressure coefficient contour plot close to the leading edge trough at $\alpha_{g}=4.82^{\circ}$ a. Red line represents the sonic line and black lines represent four streamlines. Pressure coefficient distribution close to the leading edge along the streamlines (b). Top view of streamlines coloured by Mach number

(c). Dotted black line represents the sonic line and the dashed black line represents the trough section of the convergent-divergent nozzle shape.

and, as a consequence, the benefit of the trough section decreases.

\section{Streamwise vorticity evolution}

Figure 14 and 15 analyse the evolution of streamwise vorticity inside the laminar boundary layer at $\alpha_{g}=3.00^{\circ}$ and $\alpha_{g}=6.00^{\circ}$. The streamwise vorticity has been evaluated during the post-processing, using a first order scheme to calculate the velocity derivatives. For $\alpha_{g}=3.00^{\circ}$ two streamwise vortex (SV) sheets, generated by the turning of spanwise vorticity, are observed at $x=-0.48$ on each side of the peak section. The reverse flow region at the trough generates localised streamwise vorticity $(x=-0.45)$. Vorticity sheets pairs are generated above and below the original SV sheets with an opposite sign and they develop downstream the reattachment point $(x=-0.40)$. The boundary layer is still laminar downstream the reattachment and the sheets pairs develop at a slow rate and symmetric with respect to the trough section. Streamwise vorticity appears very close to the wall, initially around the trough section $(x=-0.35)$ and then also around the peak one $(x=-0.15)$. During the downstream motion, the vorticity decay associated with the vorticity spreading is observed. The main result displayed in the streamwise vorticity flow field is the generation of vorticity sheets pairs in the trough region that energise the boundary layer without inducing transition to turbulence. As a consequence, the laminar boundary layer is three dimensional and the vorticity develops at a slow rate.

Figure 15 shows the streamwise vorticity at $\alpha_{g}=6.00^{\circ}$ in order to observe differences and similarities with the low incidence angle flow field. Streamwise vortex sheets are again generated at the leading edge, but in this case the reverse flow region is thicker and it has a greater influence on the flow field around the trough $(x=-0.45)$. Once the flow reattaches, the flow is different from the low incidence case $(x=-0.40)$. The upper and lower vorticity regions are generated again above and below the original vortex sheets but the simmetry with respect to the trough section is lost. An additional vortex pair is generated on top. Moreover, the wall vorticity is observed more upstream at higher angles of attack. Overall, the vorticity region around the trough is thicker at $\alpha_{g}=6.00^{\circ}$. Moving downstream, the vorticity decay is stronger on the upper part of the boundary layer and the pair on top becomes very weak $(x=-0.25)$. The flow field at $\alpha_{g}=6.00^{\circ}$ shows that downstream the reverse flow region the flow is still laminar and, 


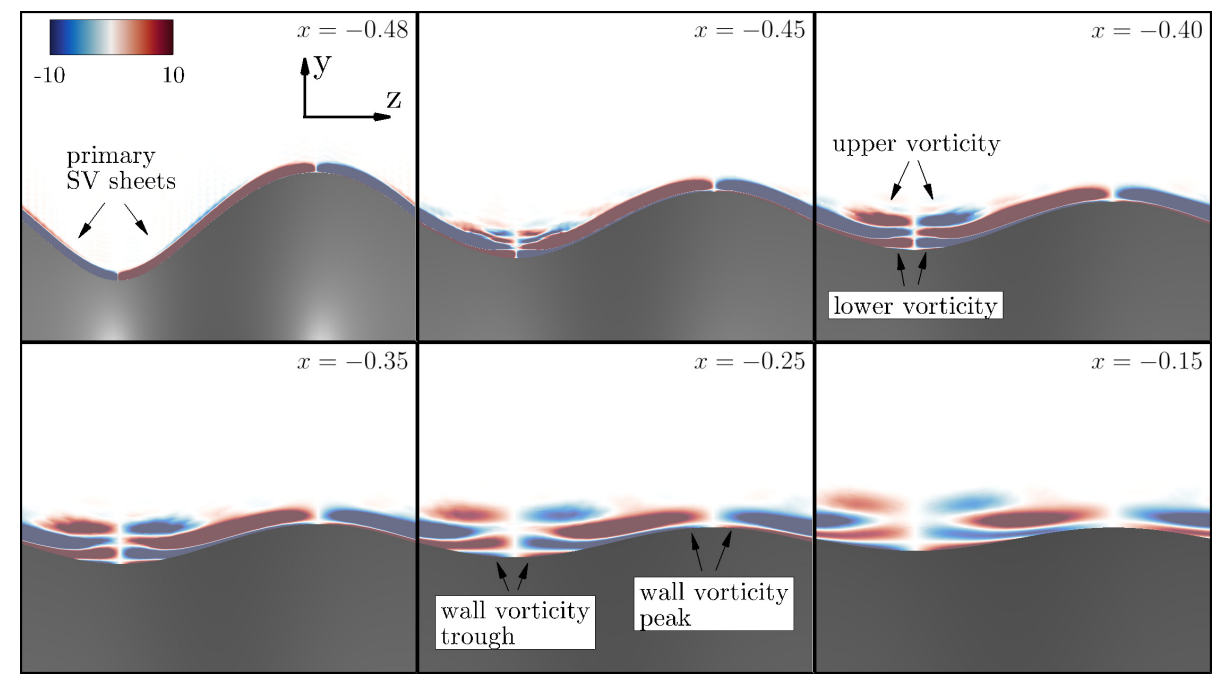

FIG. 14: Contour plots of streamwise vorticity from $x=-0.48$ to $x=-0.15$ for the wavy aerofoil at $\alpha_{g}=3.00^{\circ}$.

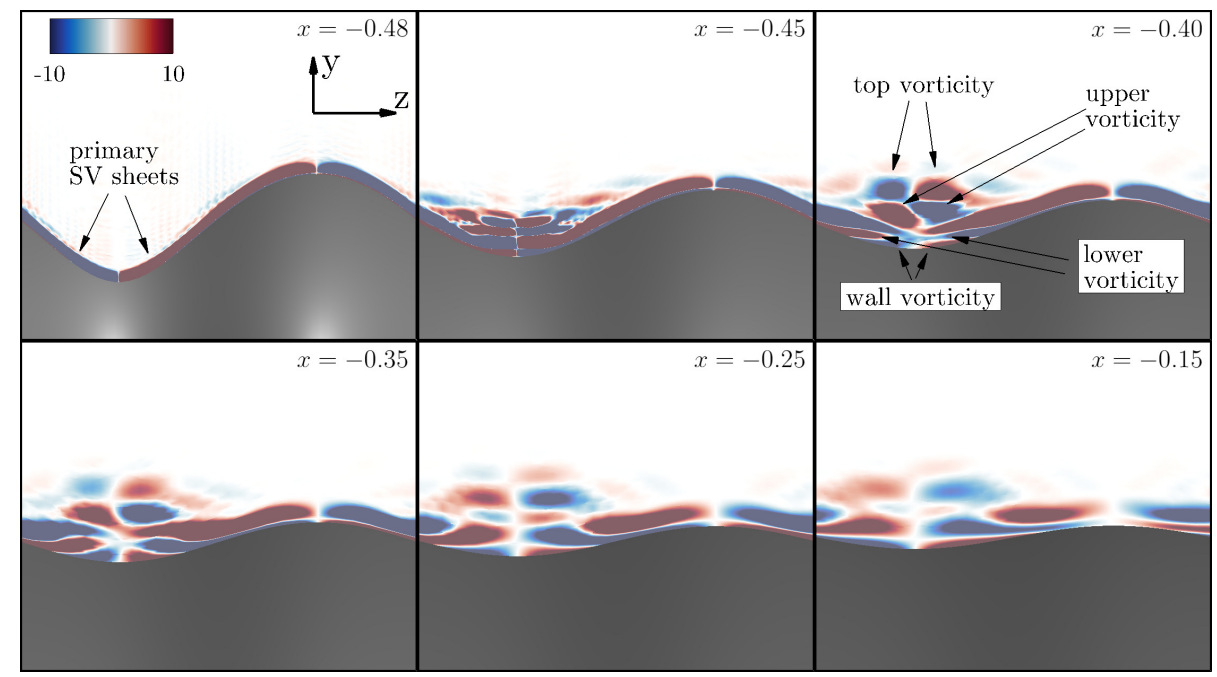

FIG. 15: Contour plots of streamwise vorticity from $x=-0.48$ to $x=-0.15$ for the wavy aerofoil at $\alpha_{g}=6.00^{\circ}$.

as a consequence, the flow development further downstream happens at a slow rate and symmetric with respect to the trough section. The streamwise vorticity field shows some differences but the main vorticity pairs are observed as in the low angle case.

\section{OBSERVATIONS ON $\lambda$-SHOCK STRUCTURE}

The previous sections have shown that the modified aerofoil introduces new flow features in the leading edge region such as streamwise vorticity and reverse flow at the trough. These have an influence on the flow downstream and this section has the objective to analyse it. Firstly, the shock waves over the baseline and the wavy aerofoils are compared for two different conditions, one with similar structures between the two aerofoils and one with different structures. Then, the condition with major differences is investigated in more details trying to relate the different shock waves structure to the upstream flow characteristics. In addition, the interaction between the shock waves and the laminar and turbulent boundary layer is analysed in further details.

At low incidence angles, weak shock waves travel upstream on the upper surface of both aerofoils, becoming weaker and finally detaching from the aerofoil at the LE. In this condition the main effect on the aerodynamic characteristics is the modified flow in the LE region, hence the shock structure is similar on both aerofoils (Figure $5 \mathrm{p}, \mathrm{d}$ ).

Increasing the angle of attack the shock waves become stronger and some differences can be observed. First of 


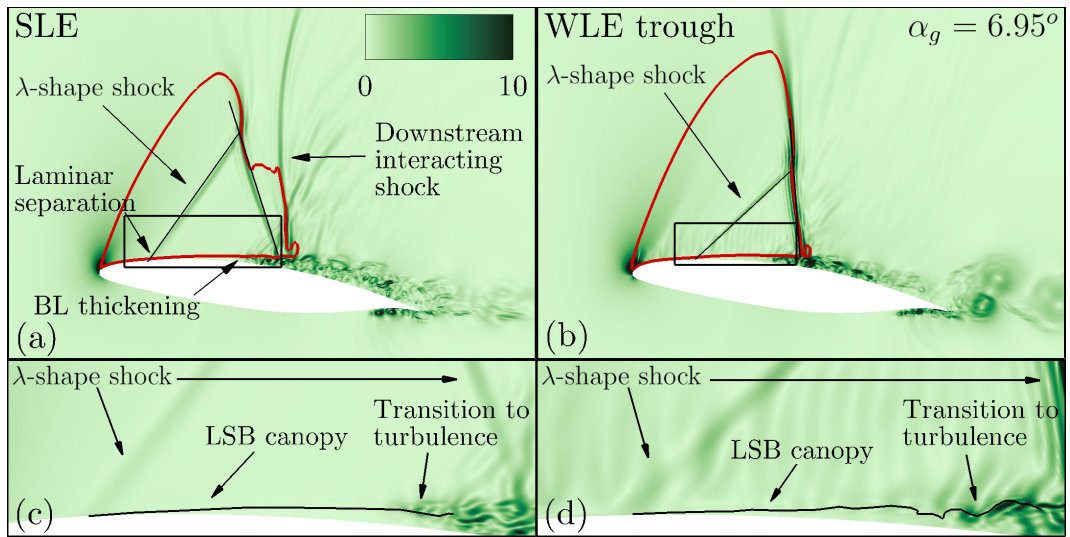

FIG. 16: Pressure gradient magnitude of SLE (a) and WLE trough (b) at $\alpha_{g}=6.95^{\circ}$. The red line highlights the sonic line and the black rectangle indicates the SBLI region plotted in (c) and (d).

all, a $\lambda$-shape shock wave is observed over the wavy aerofoil (Figure $5 \mathrm{f}$ ). This behaviour can be explained with the upstream developed streamwise vorticity which energises the BL and decreases the shock waves oscillations. On the contrary, on the straight counterpart shock waves are interacting with each other and with the BL. The consequence of these different shock shapes is a more downstream position of the laminar separation on the wavy aerofoil (Figure 5.) which has a positive effect on the lift performance (Figure 5h). This can be explained with the positive pressure gradient generated by the shock waves which tends to separate the BL. The presence of moving and interacting shock waves on the baseline aerofoil brings this positive pressure gradient upstream and causes an upstream movement of laminar separation. This intermediate condition is useful to understand the mutual interaction between shock wave structure and BL streamwise development.

At $\alpha_{g}=6.95^{\circ}$, both aerofoils show a $\lambda$-shape shock (Figure $16 \mathrm{a}, \mathrm{b}$ ). On the baseline one the $\lambda$-shape shock tends to bend upstream and to interact with another shock generated downstream by the strong pressure waves coming from the TE and the turbulent BL. Downstream the $\lambda$-shock the flow is still supersonic and it becomes subsonic downstream the interacting shock. The interaction between the $\lambda$-shape shock and the laminar boundary layer is crucial to underatand the described shock structure. The forward shock of the $\lambda$ structure separates the laminar boundary layer $\left(x_{s}=-0.34\right)$ as it is shown in Figure 16. The separated laminar BL thickens and further downstream L-T transition is observed, sligthly upstream the backward shock of the $\lambda$ structure. The separation of a $2 \mathrm{D}$ laminar BL tends to move the forward shock upstream and as a consequence, the $\lambda$-shock structure bends upstream. The result is that downstream the backward shock, the flow is still supersonic and this allows the development of the interacting normal shock which brings the flow to subsonic conditions.

On the wavy aerofoil the shock structure does not have this detrimental effect on the BL because of the streamwise vorticity generated upstream which energises the BL. The interaction between the forward shock and the laminar BL is radically changed, the positive pressure gradient separates the $\mathrm{BL}\left(x_{s}=-0.29\right)$ but there is no upstream movement of the separation point and as a consequence no bending of the $\lambda$ structure. Figure 16 $\mathrm{d}$ shows that downstream the BL separation the behaviour is similar to the straight counterpart with transition to turbulence and interaction of turbulent BL with backward shock.

On both aerofoils the backward shock of the $\lambda$ structure interacts with a turbulent BL (Figure 16a,b). Whether there is separation downstream the interaction or not, it has been observed ${ }^{41}$ a BL thickening due to the interaction with a shock wave. The thickening is higher over the baseline aerofoil for two main reasons: the turbulent BL interaction with both the backward shock and the downstream normal shock over the baseline aerofoil and the more energetic BL over the modified aerofoil. Previous studies ${ }^{41}$ show that vortex generators can be used to control the shock turbulent BL interaction and this result seems to be consistent with previous analysis. Figure 16b shows some vortices detaching from the aerofoil. They are related to the pressure difference between the upper and the lower surface at the TE.

To sum up, the SBLI over the V2C aerofoil at high angles of attack in transonic flow is characterised by a $\lambda$-shape shock, where the forward shock interacts with a laminar BL and the backward one interacts with a turbulent BL. The driving mechanism is the laminar BL separation due to the interaction with the forward shock. An upstream movement of the laminar separation can cause a bending of the $\lambda$-shape shock and the generation of an additional normal shock downstream. This can have an impact on the interaction with the turbulent BL, which can thickens because of a stronger interaction with two shocks. The generation of three dimensional laminar boundary layer and streamwise vorticity at the trough of the modified aerofoil is crucial to control the laminar BL separation and avoid 
its upstream movement.

This different SBLI at high angles can have an important impact on the flow unsteadiness. Previous studies ${ }^{39}$ show that at $\alpha=7.0^{\circ}$ the flow is characterised by the shock buffet phenomenon. The more energetic laminar BL over the wavy aerofoil can have a major impact on the control of shock waves oscillation and should be analysed in further details with a static analysis.

\section{CONCLUDING REMARKS AND FUTURE WORK}

The wavy leading edges over laminar supercritical aerofoils in transonic flow show promising results. This preliminary study has analysed the performance of the modified aerofoil at different angles of attack through a heaving motion. The main focus of the paper has been the quasi-linear part of the heaving motion. In the first part, the aerodynamic performance has been studied in details both in chordwise and spanwise directions. The main results are summarised as follows.

- The wavy geometry generates a distortion of the flow field in the leading edge region and this has an impact on the transition point which is moved upstream. The LE distortion enhances the disturbances initial size and growth.

- The drag coefficient is decreased in two main regions at low and moderate angles of attack: the leading edge and downstream the transition point. Increasing the angle of attack, the beneficial effect of the leading edge is decreased and the second region becomes dominant for the aerofoil performance.

The second part has analysed the flow field at the main sections of the wavy aerofoil (peak, hill and trough) and compared it with the baseline case. The leading edge analysis allows to explain the drag reduction at the LE region. The flow channeling phenomenon explains the acceleration to supersonic conditions of the flow at the trough in a wide range of angles of attack, which has an influence on the enhancement of the lift coefficient. The boundary layer and the streamwise vorticity evolution show that the modified leading edge energises the laminar boundary layer and makes it three dimensional.

In the third part, the results obtained during the previous analysis are exploited to explain the shock waves structure. At low incidence angles, the shock structure is similar over the two aerofoils and the aerodynamic characteristics are driven by the LE flow behaviour. A significant difference can be noticed at high angles of attack. The baseline aerofoil is characterised by the interaction of the main $\lambda$-shape shock with a downstream shock due to an upstream movement of the laminar separation, which modifies the shape of the $\lambda$ shock and thickens the BL. The wavy aerofoil shows a different shock boundary layer interaction without the bending of the $\lambda$-shape shock due to a more energetic three dimensional laminar BL, which can be crucial for the control of shock buffet phenomenon.

The dynamic motion of the two aerofoils will be investigated in details in the next study, showing the effect of WLEs on the control of added mass effects. The wavy geometry is beneficial in low-speed flows to control the stall condition and the pressure waves emission in the far field. An unsteady analysis of the aerofoil requires a static condition. The future works aim also to extend the performance analysis of the wavy aerofoil in transonic flow at high angles of attack $\left(\alpha=6^{\circ}-7^{\circ}\right)$ and to study the control of the shock buffet phenomenon. This should be followed by an aeroacoustic analysis in order to understand how these unsteady phenomena propagate in the far field.

\section{ACKNOWLEDGEMENTS}

The authors gratefully acknowledge the support of EPSRC (Engineering and Physical Sciences Research Council) for the present work under the CDT (Centre for Doctoral Training) in NGCM (Next Generation Computational Modelling) at the University of Southampton. We also acknowledge the high-performance computing facilities and services of the UK National Supercomputer ARCHER and the local IRIDIS5 at the University of Southampton in the completion of this work. We thank Dassault Systèmes for providing us the V2C laminar supercritical aerofoil profile.

\section{Appendix A: Full heaving motion}

The aim of this section is to illustrate the aerodynamic characteristics of the full heaving cycle. Figure 17 shows the lift and drag coefficients. The lift coefficient drops abruptly for the baseline case when the geometric angle of attack decreases. This is related to a flow separation; in high speed flows previous studies prove that the presence of a shock wave in the suction side of the aerofoil leads to an abrupt thickening and subsequent separation of the boundary layer 


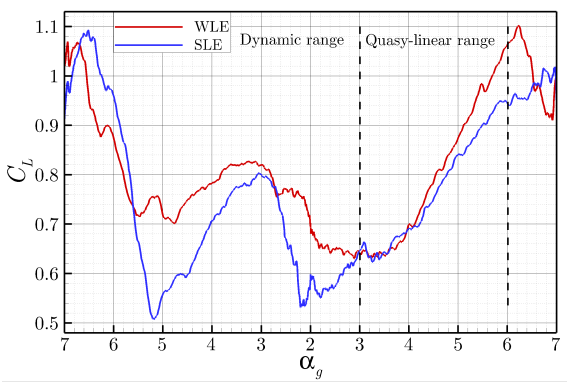

(a)

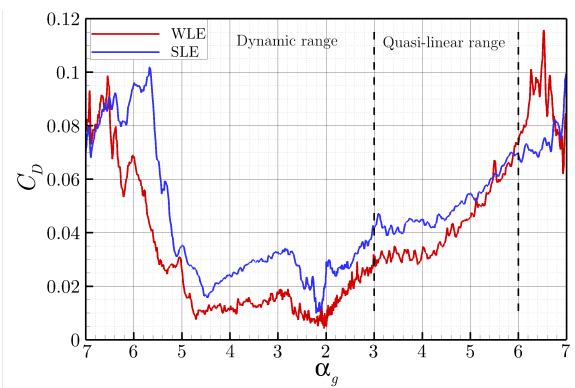

(b)

FIG. 17: Lift a and drag (b) coefficients varying the angle of attack during the full heaving period.

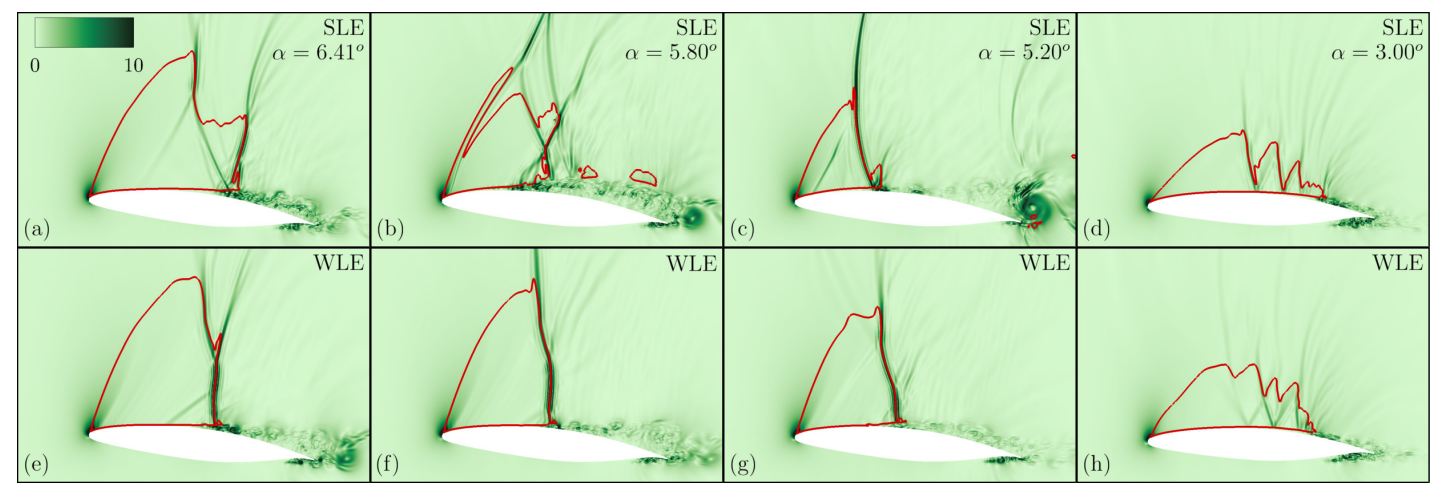

FIG. 18: Pressure gradient magnitude of SLE (a-d) and WLE (e-h) during the lift sudden drop and recovery.

flow 22143 . Over a laminar supercritical aerofoil there is a laminar separation which generates a laminar bubble and then a transition close to the shock interaction (Figure 18k). During the drop there is a sudden upstream movement of the transition point which drastically affects the shock structure. Large vortical structures are generated during this phase (Figure 18b,c). Once the shock reaches the most upstream position and the large vortices pass through the TE (Figure 18c), the lift coefficient starts recovering. The modified aerofoil shows a different behaviour, there is a sudden drop of the lift but it is related to a sudden upstream movement of the shock wave. However, the shock wave shows less oscillations and the interaction with the boundary layer does not generate large vortical structures (Figure 18,f). The result is a moderate drop. Once the shock wave reaches the most upstream position, the recovery starts like in the baseline case (Figure $18 \mathrm{~g}$ ). The reason for the difference in the drop and recovery phases is that, even if both aerofoils show $\lambda$-shape shock at high angles, an higher strength of the leading shock on the baseline aerofoil is observed together with a more upstream location of the interaction with the boundary layer. The shock wave generates a positive pressure gradient which tends to destabilise the laminar boundary layer. As a consequence, the stronger leading shock on the SLE generates a larger BL separation. The result on the drag (Figure 17b is that the drag coefficient of the wavy aerofoil is lower during the separation and recovery phases and thus the efficiency is higher.

The analysis of the dynamic range of the heaving motion shows that the separation on the suction side of the straight aerofoil is driven by the sudden upstream movement of the transition point which modifies the shock structure and generates large vortices. On the other hand, the separation over the wavy aerofoil is driven by the upstream movement of the shock wave due to its increased stability.

\section{DATA AVAILABILITY}

All data supporting this study are openly available from the University of Southampton repository at http://dx.doi.org/10.5258/SOTON/XXXXXX.

\footnotetext{
${ }^{1}$ M. Iovnovich and D. Raveh, "Transonic unsteady aerodynamics in the vicinity of shock-buffet instability," Journal of Fluids and Structures 29, 131-142 (2012).
} 
${ }^{2}$ C. Gao, W. Zhang, and Z. Ye, "Numerical study on closed-loop control of transonic buffet suppression by trailing edge flap," Computers \& Fluids 132, 32-45 (2016).

${ }^{3}$ D. Caruana, A. Mignosi, M. Corrège, A. Le Pourhiet, and A. Rodde, "Buffet and buffeting control in transonic flow," Aerospace Science and Technology 9, 605-616 (2005).

${ }^{4}$ M. Rybalko, H. Babinsky, and E. Loth, "Vortex generators for a normal shock/boundary layer interaction with a downstream diffuser," Journal of Propulsion and Power 28, 71-82 (2012).

${ }^{5}$ N. Titchener and H. Babinsky, "Shock wave/boundary-layer interaction control using a combination of vortex generators and bleed," AIAA Journal 51, 1221-1233 (2013).

${ }^{6} \mathrm{H}$. A. Holden and H. Babinsky, "Effect of microvortex generators on seperated normal shock/boundary layer interactions," Journal of Aircraft 44, 170-174 (2007).

${ }^{7}$ J. Huang, Z. Xiao, J. Liu, and S. Fu, "Simulation of shock wave buffet and its suppression on an oat15a supercritical airfoil by iddes," Science China Physics, Mechanics and Astronomy 55, 260-271 (2012).

${ }^{8}$ P. Molton, J. Dandois, A. Lepage, V. Brunet, and R. Bur, "Control of buffet phenomenon on a transonic swept wing," AIAA Journal 51, 761-772 (2013).

${ }^{9}$ G. Godard and M. Stanislas, "Control of a decelerating boundary layer. part 1: Optimization of passive vortex generators," Aerospace Science and Technology 10, 181-191 (2006).

${ }^{10}$ S. Koike, K. Nakakita, T. Nakajima, S. Koga, M. Sato, H. Kanda, K. Kusunose, M. Murayama, Y. Ito, and K. Yamamoto, "Experimental investigation of vortex generator effect on two-and three-dimensional nasa common research models," in 53rd AIAA Aerospace Sciences Meeting (2015) p. 1237.

${ }^{11}$ Y. Ito, K. Yamamoto, K. Kusunose, S. Koike, K. Nakakita, M. Murayama, and K. Tanaka, "Effect of vortex generators on transonic swept wings," Journal of Aircraft 53, 1890-1904 (2016).

${ }^{12}$ E. Stanewsky, J. Délery, J. Fulker, and W. Geißler, EUROSHOCK-Drag Reduction by Passive Shock Control: Results of the Project EUROSHOCK, AER2-CT92-0049 Supported by the European Union, 1993-1995, Vol. 56 (Springer Science \& Business Media, 2013).

${ }^{13}$ J. Birkemeyer, H. Rosemann, and E. Stanewsky, "Shock control on a swept wing," Aerospace Science and Technology 4, 147-156 (2000).

${ }^{14}$ B. König, M. Pätzold, T. Lutz, E. Krämer, H. Rosemann, K. Richter, and H. Uhlemann, "Numerical and experimental validation of three-dimensional shock control bumps," Journal of Aircraft 46, 675-682 (2009).

${ }^{15}$ M. Pätzold, T. Lutz, E. Kramer, and S. Wagner, "Numerical optimization of finite shock control bumps," in 44th AIAA Aerospace Sciences Meeting and Exhibit (2006) p. 1054.

${ }^{16}$ J. P. Eastwood and J. P. Jarrett, "Toward designing with three-dimensional bumps for lift/drag improvement and buffet alleviation," AIAA Journal 50, 2882-2898 (2012).

${ }^{17}$ R. Mayer, T. Lutz, E. Krämer, and J. Dandois, "Control of transonic buffet by shock control bumps on wing-body configuration," Journal of Aircraft 56, 556-568 (2019).

${ }^{18}$ P. Bruce and S. Colliss, "Review of research into shock control bumps," Shock Waves 25, 451-471 (2015).

${ }^{19}$ S. Bogdanski, K. Nübler, T. Lutz, and E. Krämer, "Numerical investigation of the influence of shock control bumps on the buffet characteristics of a transonic airfoil," in New results in numerical and experimental fluid mechanics IX (Springer, 2014 ) pp. 23-32.

${ }^{20}$ R. Pérez-Torró and J. W. Kim, "A large-eddy simulation on a deep-stalled aerofoil with a wavy leading edge," Journal of Fluid Mechanics 813, 23-52 (2017).

${ }^{21}$ K. L. Hansen, N. Rostamzadeh, R. M. Kelso, and B. B. Dally, "Evolution of the streamwise vortices generated between leading edge tubercles," Journal of Fluid Mechanics 788, 730-766 (2016).

${ }^{22}$ L. Siconolfi, S. Camarri, and J. H. Fransson, "Boundary layer stabilization using free-stream vortices," Journal of Fluid Mechanics $\mathbf{7 6 4}$ (2015).

${ }^{23}$ N. Rostamzadeh, K. Hansen, R. Kelso, and B. Dally, "The formation mechanism and impact of streamwise vortices on naca 0021 airfoil's performance with undulating leading edge modification," Physics of Fluids 26, 107101 (2014).

${ }^{24}$ D. Custodio, C. Henoch, and H. Johari, "Aerodynamic characteristics of finite span wings with leading-edge protuberances," AIAA Journal 53, 1878-1893 (2015).

${ }^{25}$ H. Yoon, P. Hung, J. Jung, and M. Kim, "Effect of the wavy leading edge on hydrodynamic characteristics for flow around low aspect ratio wing," Computers \& Fluids 49, 276-289 (2011).

${ }^{26}$ F. M. White, Viscous Fluid Flow (McGraw-Hill, 1991).

${ }^{27}$ J. W. Kim and P. J. Morris, "Computation of subsonic inviscid flow past a cone using high-order schemes," AIAA Journal 40, 1961-1968 (2002).

${ }^{28}$ J. W. Kim, A. S. H. Lau, and N. D. Sandham, "CAA boundary conditions for airfoil noise due to high-frequency gusts," Procedia Engineering 6, 244-253 (2010).

${ }^{29}$ J. W. Kim, A. S. H. Lau, and N. D. Sandham, "Proposed boundary conditions for gust-airfoil interaction noise," AIAA Journal 48, 2705-2709 (2010).

${ }^{30}$ J. W. Kim, "Optimised boundary compact finite difference schemes for computational aeroacoustics," Journal of Computational Physics 225, 995-1019 (2007).

${ }^{31}$ J. W. Kim, "High-order compact filters with variable cut-off wavenumber and stable boundary treatment," Computers \& Fluids 39, $1168-1182(2010)$.

${ }^{32}$ J. W. Kim and D. J. Lee, "Adaptive nonlinear artificial dissipation model for computational aeroacoustics," AIAA Journal 39, 810-818 (2001).

${ }^{33}$ J. W. Kim and D. J. Lee, "Generalized characteristic boundary conditions for computational aeroacoustics," AIAA Journal 38, 2040-2049 (2000).

${ }^{34}$ J. W. Kim and D. J. Lee, "Generalized characteristic boundary conditions for computational aeroacoustics, part 2," AIAA Journal 42, 47-55 (2004).

${ }^{35}$ J. W. Kim, "Quasi-disjoint pentadiagonal matrix systems for the parallelization of compact finite-difference schemes and filters," Journal of Computational Physics 241, 168-194 (2013).

${ }^{36}$ A. Skillen, A. Revell, A. Pinelli, U. Piomelli, and J. Favier, "Flow over a wing with leading-edge undulations," AIAA Journal 53, 464-472 (2015).

${ }^{37}$ D. J. Garmann, M. R. Visbal, and P. D. Orkwis, "Comparative study of implicit and subgrid-scale model large-eddy simulation techniques for low-reynolds number airfoil applications," International Journal for Numerical Methods in Fluids 71, 1546-1565 (2013). 
${ }^{38}$ K. A. Hoffmann and S. T. Chiang, Computational Fluid Dynamics, 4th ed., Vol. 2 (Engineering Education System, 2000 ) pp. $21-31$.

${ }^{39}$ D. Szubert, I. Asproulias, F. Grossi, R. Duvigneau, Y. Hoarau, and M. Braza, "Numerical study of the turbulent transonic interaction and transition location effect involving optimisation around a supercritical aerofoil," European Journal of Mechanics-B/Fluids 55, 380-393 (2016).

${ }^{40}$ J. Dandois, I. Mary, and V. Brion, "Large-eddy simulation of laminar transonic buffet," Journal of Fluid Mechanics 850, 156-178 (2018).

${ }^{41}$ J. M. Delery, "Shock wave/turbulent boundary layer interaction and its control," Progress in Aerospace Sciences 22, 209-280 (1985).

${ }^{42}$ T. C. Corke and F. O. Thomas, "Dynamic stall in pitching airfoils: aerodynamic damping and compressibility effects," Annual Review of Fluid Mechanics 47, 479-505 (2015).

${ }^{43}$ M. Chandrasekhara, M. Wilder, and L. Carr, "Competing mechanisms of compressible dynamic stall," AIAA Journal 36, 387-393 (1998). 